KINEMATIC EVALUATION OF END EFFECTOR DESIGN

by

Gary Wayne Edwards

B.S. Physics/Mathematics

University of Richmond (1979)

Submitted in partial fulfillment of the

requirements for the dual degrees of

OCEAN ENGINEER

and

MASTER OF SCIENCE IN OCEAN ENGINEERING

at the
MASSACHUSETTS INSTITUTE OF TECHNOLOGY
and the
WOODS HOLE OCEANOGRAPHIC INSTITUTION

September 1992

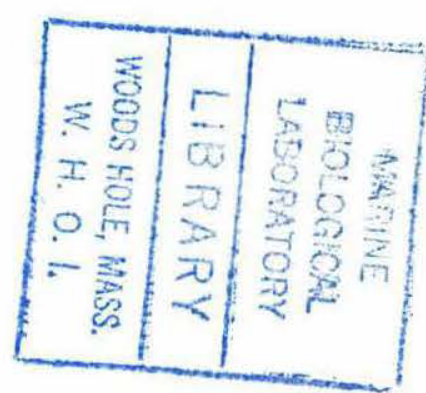

(c) Gary Wayne Edwards

The author hereby grants to MIT and WHOI permission to reproduce and distribute copies of this thesis document in whole or in part.

Signature of Author.

Joint Program in Applied Ocean Science and Engineering Massachusetts Institute of Technology

Woods Hole Oceąnographic Instifution

Certified by

Dr. Nathan T. Ulrich

Assistant Scientist

Woods Hole Oceanographic Institution

Thesis Supervisor

Acceptedby

Dr. Arthur B. Baggeroer Chairman, Joint Committee for Applied Ocean Science and Engineering

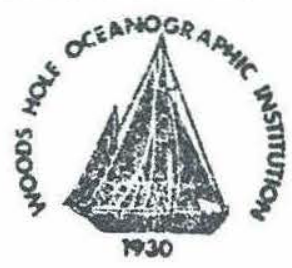

Massachusetts Institute of Technology/

Woods Hole Oceanographic Institute 


\title{
KINEMATIC EVALUATION OF END EFFECTOR DESIGN
}

\author{
by \\ GARY WAYNE EDWARDS \\ Submitted to the Massachusetts Institute of Technology/ \\ Woods Hole Oceanographic Institution \\ Joint Program in Ocean Engineering \\ in August 1992 in partial fulfillment of the \\ requirements for the degrees of \\ OCEAN ENGINEER \\ and \\ MASTER OF SCIENCE IN OCEAN ENGINEERING
}

\begin{abstract}
The complex, many degree-of-freedom end effectors at the leading edge of technology would be unusable in the sea bottom research environment. Simpler designs are required to provide adequate reliability for subsea use. This work examines selection of end effector designs to achieve optimum grasping ability with minimal mechanical complexity. A new method of calculating grasp stability is developed, incorporating elements of previous works in the field. Programs are developed which evaluate the ability of different end effector configurations to grasp representative objects (a cube, sphere and infinite cylinder). End effector designs considered had circular palms with fingers located at the periphery, oriented so that each pointed to the center of the palm. The program tested configurations of from 1 to 4 fingers and from 1 to 3 links per finger. Three sets of finger proportions were considered: equal length links, half length links, and anthropomorphic proportions. The 2 finger, 2 link per finger configuration was determined to be the optimum design, and the half length proportions were selected as the best set of proportions.
\end{abstract}

Thesis Supervisor:

Dr. Nathan T. Ulrich

Assistant Scientist

Woods Hole Oceanographic Institution 


\section{Contents}

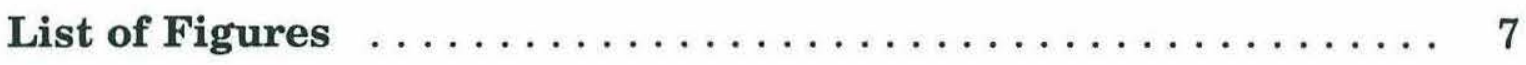

Chapter 1 Introduction $\ldots \ldots \ldots \ldots \ldots \ldots \ldots \ldots \ldots \ldots, 9$

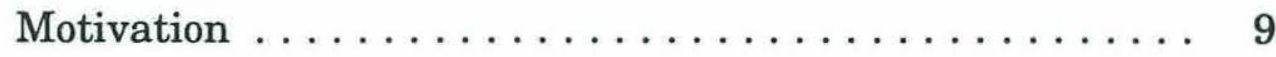

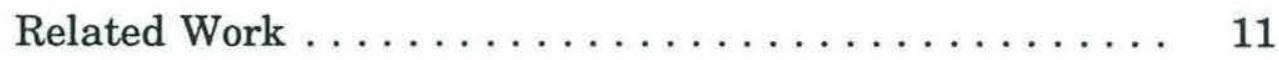

Notation and Definitions $\ldots \ldots \ldots \ldots \ldots \ldots \ldots \ldots$

Thesis Outline $\ldots \ldots \ldots \ldots \ldots \ldots \ldots \ldots \ldots \ldots \ldots \ldots$

Chapter 2 The Stability Algorithm $\ldots \ldots \ldots \ldots \ldots \ldots \ldots$

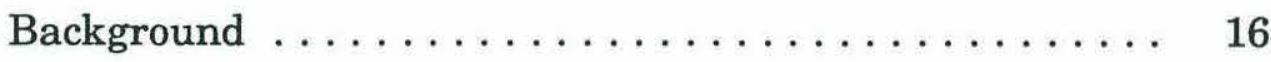

Dealing with Friction $\ldots \ldots \ldots \ldots \ldots \ldots \ldots \ldots \ldots$

Virtual Work $\ldots \ldots \ldots \ldots \ldots \ldots \ldots \ldots \ldots \ldots \ldots \ldots \ldots \ldots \ldots$

Mathematics Used in the Stability Algorithm . . . . . . . 21

Conceptual Examples of Stability Determination ...... 25

Characterization of Stability Criteria: Form or Force

Closure? . . . . . . . . . . . . . . . . . . $\quad 29$

Chapter 3 The Grasp Tester $\ldots \ldots \ldots \ldots \ldots \ldots \ldots \ldots \ldots \ldots \ldots$

Background on Grasp Tester $\ldots \ldots \ldots \ldots \ldots \ldots \ldots . \quad 32$

Hand Assumptions for the Grasp Tester $\ldots \ldots \ldots \ldots .32$

Object Selection $\ldots \ldots \ldots \ldots \ldots \ldots \ldots \ldots \ldots \ldots \ldots \ldots \ldots$ 


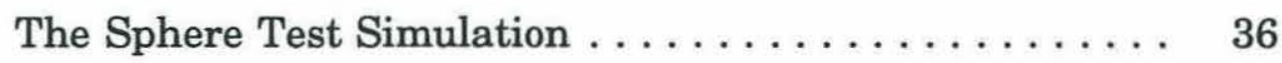

The Cylinder Tester . . . . . . . . . . . . 38

The Cube Tester $\ldots \ldots \ldots \ldots \ldots \ldots \ldots \ldots \ldots \ldots \ldots$

Scoring and Evaluation $\ldots \ldots \ldots \ldots \ldots \ldots \ldots \ldots \ldots$

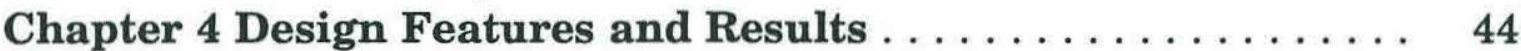

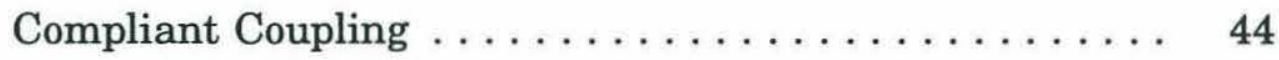

Palm Prehension $\ldots \ldots \ldots \ldots \ldots \ldots \ldots \ldots \ldots \ldots \ldots$

Finger Length and Proportion $\ldots \ldots \ldots \ldots \ldots \ldots \ldots$

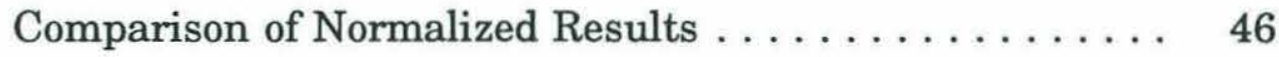

Finger Proportions $\ldots \ldots \ldots \ldots \ldots \ldots \ldots \ldots \ldots \ldots$

Discussion of Individual Tests $\ldots \ldots \ldots \ldots \ldots \ldots \ldots \quad 54$

Sphere Tests $\ldots \ldots \ldots \ldots \ldots \ldots \ldots \ldots \ldots 4$

The Cube Tests $\ldots \ldots \ldots \ldots \ldots \ldots \ldots \ldots \ldots$

The Cylinder Tests . . . . . . . . . . . 57

The $2 / 2$ Half Length Configuration $\ldots \ldots \ldots \ldots \ldots \ldots \ldots .60$

Chapter 5 Model Evaluation and Conclusion $\ldots \ldots \ldots \ldots \ldots 61$

Model Validation $\ldots \ldots \ldots \ldots \ldots \ldots \ldots \ldots \ldots \ldots \ldots \ldots$

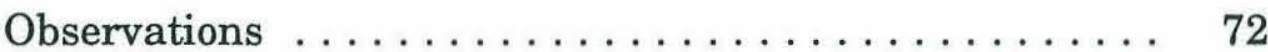

Appendix 1: Grasp Tester Results $\ldots \ldots \ldots \ldots \ldots \ldots \ldots \ldots 78$

Grasp Tester Results for Half Length Proportions . . . . . . . 79

Grasp Tester Results for Equal Length Proportions . . . . . . 80 
Grasp Tester Results for Anthropomorphic Proportions . . . . . 81

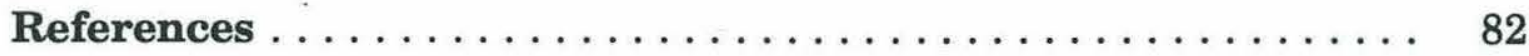




\section{List of Figures}

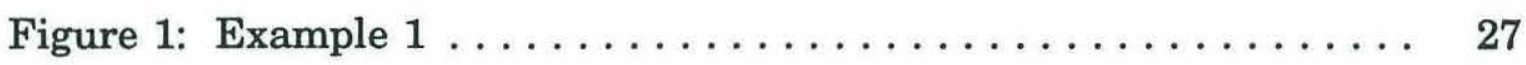

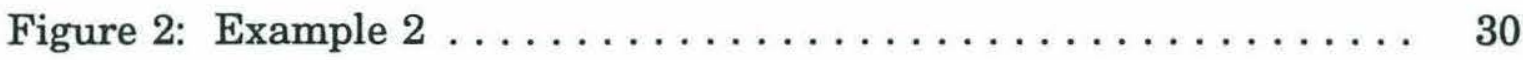

Figure 3: Allowed and disallowed finger positions $\ldots \ldots \ldots \ldots \ldots 34$

Figure 4: The Jason/Medea vehicle system $\ldots \ldots \ldots \ldots \ldots \ldots \ldots .35$

Figure 5: Normalized results for equal proportions $\ldots \ldots \ldots \ldots \ldots 47$

Figure 6: Normalized results for half length proportions . . . . . . . 48

Figure 7: Normalized results for anthropomorphic proportions . . . 49

Figure 8: Total scores for equal length proportions $\ldots \ldots \ldots \ldots \ldots 51$

Figure 9: Total score for half length proportions $\ldots \ldots \ldots \ldots \ldots \ldots 2$

Figure 10: Total scores for anthropomorphic proportions . . . . . . 53

Figure 11: 2 link/half length and 3 link/equal length geometry identical

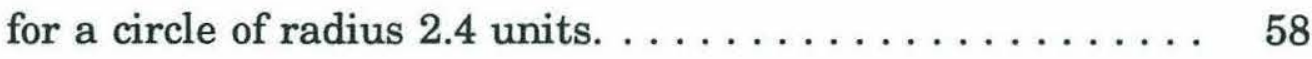

Figure 12: Location of base joints for 3 finger configuration limits wrapping ability compared to 2 finger configuration $\ldots \ldots \quad 59$

Figure 13: Validation model showing $4 / 2$ configuration $\ldots \ldots \ldots \ldots 62$

Figure 14: Stable grasp on a sphere with a 2/2 configuration end

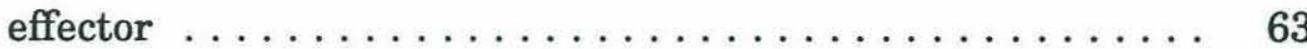


Figure 15: Unstable grasp on a sphere by a $2 / 2$ configuration end

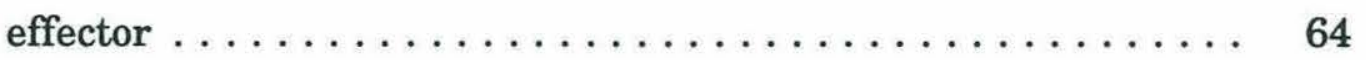

Figure 16: Sphere position test with 2/2 configuration end effector $\ldots \ldots \ldots \ldots \ldots \ldots \ldots \ldots \ldots \ldots \ldots \ldots \ldots \ldots \ldots$

Figure 17: Sphere position test with 4/2 configuration end effector, fourth finger removed for clarity $\ldots \ldots \ldots \ldots \ldots \ldots 67$

Figure 18: Stable grasp on a cylinder by a $3 / 2$ end effector $\ldots \ldots \ldots 68$

Figure 19: Stable grasp on a cylinder by a $2 / 2$ end effector $\ldots \ldots \ldots 69$

Figure 20: Unstable cylinder position test for a $2 / 2$ end effector $\ldots \ldots 70$

Figure 21: Comparison of wrapping abilities for 2 and 3 link fingers of

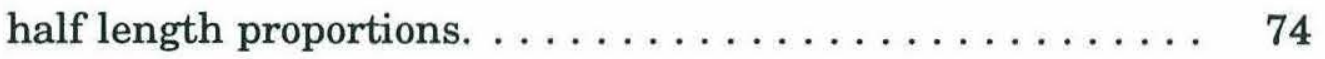

Figure 22: Methods of enhancing sphere stability: a) Finger shape,

b) Configuration and orientation $\ldots \ldots \ldots \ldots \ldots \ldots \ldots \quad 76$ 


\section{Chapter 1}

\section{Introduction}

\section{Motivation}

Much work has been done in the field of end effector development, mostly dealing with high dexterity manipulation in the laboratory environment. The result is often impressive, typically complex, but unfortunately often suitable for use only in the research laboratory. Our purpose is different; we want to design a useful and reliable end effector that will function adequately in a tele-robotic application at the bottom of the ocean. Many desirable characteristics of a research end effector become liabilities at deep submergence depths. A highly dexterous end effector may be unreliable underwater. Our requirements demand adequate dexterity for deep sea research tasks along with the simplicity required for reliability in this harsh environment.

Commercially available deep sea end effectors are predominantly two fingered, single degree of freedom devices. In general, they can be grouped into lobster claw-like manipulators and parallel jaw grippers. This is far removed from the state of the art in research end effectors, probably for the 
reasons cited above. Our goal is an end effector with more capability than current designs that still works reliably subsea. The primary design question then becomes, "What finger configuration or degree of mechanical complexity is appropriate given these limitations?" In an effort to answer this question, we have written a simulation program that tests the grasp of various hand configurations on several representative objects. The results allow us to select a hand configuration with maximum grasp capability for minimum mechanical complexity.

Several assumptions are made about the hand and fingers to simplify the process and limit consideration to reasonable designs. We will include aspects of mechanical intelligence as proposed by Ulrich [Ulrich 88], especially the idea of compliant coupling between finger joints. The attraction of compliant coupling is two-fold; its use can reduce the number of required actuators as well as allow design of a hand which automatically conforms to an object so that computer generated grip planning algorithms are not required. As early as 1977, Hanafusa and Asada's work indicated that compliant coupling between fingers enhanced grasping ability for a single actuator, multi-fingered hand [Hanafusa 77] $]^{1}$.

Also from Ulrich, all designs considered will incorporate the concept of palm prehension. This simply means the hand includes a palm that will be

1. This was not one of Hanafusa and Asada's conclusions, it is the author's observation based on the results contained in their paper. 
intentionally utilized in grasping, which provides the capability of 1 or more additional contact points with no increase in the number of actuators required. The inclusion of these "free" contacts increases the probability of achieving a stable grasp for an arbitrary object. A necessary (but not sufficient) condition for stable grasping of a general 6 degree-of-freedom object with unidirectional contact forces is that there be at least 7 contact wrenches [Lakshminarayana 78]. If we consider frictional forces, then each contact gives 3 orthogonal contact wrenches. Designing for palm prehension can allow us to achieve stability with 1 or 2 less fingers than otherwise would be required.

A major design goal was inclusion of the capability to stably grasp a variety of marine objects, biological samples and hydrographic instruments. Manipulation of the object being grasped was not required, manipulation here meaning the ability to transfer the object from one stable grasp to another without setting it down. Accordingly, we use a grasp testing routine which tests only for grasp stability. An alternative to this would be a connectivity and mobility analysis as done by Salisbury [Salisbury 85]. As discussed below, Salisbury's requirement was for manipulation of objects, which differs considerably from our goal. The stable grasp and no manipulation requirement simplifies hand designs under consideration.

\section{Related Work}

Much of the previous work dealt with computerized grasp planning. 
Although my purposes were different, I was influenced significantly by the work of Salisbury [Salisbury 85], which considers optimum finger and link configuration for dexterous manipulation. Salisbury examined various finger configurations numerically and determined which could give the required capabilities. He did this using a connectivity and mobility analysis, appropriate in this case because his purpose was to design a hand that was capable of fine manipulation of objects.

Ulrich introduced the medium complexity end effector as a more economical, light weight, and compact alternative to complex hands. $\mathrm{He}$ introduced the term mechanical intelligence to represent the use of mechanical design features which simplify potentially complex end effectors. Palm prehension and compliant coupling between finger joints were examples of mechanical intelligence incorporated in the Penn Hand [Ulrich 88].

Hanafusa and Asada studied stable grasping of two dimensional objects by a hand with elastic fingers. They did this by developing a potential function and defining a stable grasp as one which minimizes this function. Their experimental verification included tests of various coupling configurations between actuators and fingers. The proposed end effector used one motor driving three fingers by compliant coupling, although the experimental apparatus used three actuators driven in unison. Of particular relevance is the implicit point that inclusion of compliant coupling (in this case conventional springs) enhanced grasp stability [Hanafusa 77]. 
Ohwovoriole and Roth made major contributions to the theory of grasp stability [Ohwovoriole 81, 87]. Although screw and wrench notation and reciprocal screw and wrench pairs were well understood [Hunt 78], Ohwovoriole and Roth extended the theory to include repelling and contrary screw/wrench pairs. They used the sign of virtual work for a given grasp to determine grasp stability. These terms will be fully explained in Chapter 2 .

Nguyen used the mathematical tools of Ohwovoriole to develop algorithms capable of constructing stable grasps in both two and three dimensions on arbitrary objects[Nguyen $86,87 \mathrm{a}, 87 \mathrm{~b}$ ]. He also provided a concise summary of the concept of form closure; a grasp is form closure if the grasped object is totally constrained by the set of contacts, irrespective of the magnitude of contact forces [Nguyen 87a]. This is contrasted with force closure, which describes an object in equilibrium due to contributions of contact forces, frictional forces and possibly external forces. Nguyen observed that form closure could be viewed as force closure with frictionless point contacts only.

Greiner developed an end effector composed of three serial finger links using compliant coupling similar to that introduced by Ulrich. This end effector relied on 1 single-actuator finger which passively wrapped around objects to achieve stable grasps [Greiner 90].

Trinkle studied various aspects of grasping. He developed strategies to lift an "slippery" object away from a supporting surface, requiring prediction 
of object motion during manipulation, and used these as a component of a grasping plan for enveloping grasps [Trinkle 87].

\section{Notation and Definitions}

Several definitions and notational conventions are used in this work. Configuration is used to refer to a specific combination of number of fingers and number of joints per finger. Proportion refers to a specific set of link lengths for a multi-jointed finger. The notation $3 / 2$ denotes an end effector configuration made up of 3 fingers of 2 links per finger. The term interior intersection is used to represent an intersection between finger and grasped object which does not occur at an edge or tangent point; it results because the finger is not long enough to reach the tangent or edge intersection.

\section{Thesis Outline}

The organization of this thesis follows the major subdivisions of the work required for its completion. This chapter explains the motivation for this work, describes the basic methods used to approach the problem and discusses related works on the topic. Chapter 2 details the stability algorithm consisting of concepts from several previous works melded together to handle point contacts with friction. Chapter 3 describes the simulation which performs the kinematics for contact wrench determination on tested grasps. Chapter 4 discusses results of the grasp testing simulation and recommends a best choice 
of finger/link configuration. Chapter 5 covers validation work done with a mock up end effector which can perform the grasps evaluated by the simulation. 


\section{Chapter 2}

\section{The Stability Algorithm}

\section{Background}

Our goal is to test various hand configurations to determine an optimal configuration for stable grasping; thus we must devise a method of determining grasp stability. Many researchers have developed grasp stability algorithms for use in grasp synthesis. Our needs are different since we must close a simulated hand on an object and determine if the grasp is stable. We can determine the points of contact using kinematic principles, but then we must judge whether the resulting grasp is stable. For reasons discussed below, we found it prudent to consider point contacts with friction.

The most convenient way to describe these contacts is to use screw and wrench notation, which is explained the works of Ball and Hunt [Ball 00, Hunt 70]. Ohwovoriole defines complete constraint as grasping the object so that it can have no motions relative to the locked finger joints [Ohwovoriole 87]. Lakshminarayana noted that seven unidirectional point contacts are required to completely constrain a 6 degree-of-freedom object [Lakshminarayana 78]. Unidirectional means the contacts can push but not pull. Since we assume only one contact per link or digit, requiring 7 frictionless point contacts could 
drive eligible hand designs to an excessive level of complexity: a minimum of two 3-jointed fingers or three 2-jointed fingers (plus the palm). Intuitively, we felt that adequate functionality could be achieved with a much simpler design, so we included friction in the analysis. If frictional forces are considered, the 7 contact forces necessary for complete immobilization could be obtained from only 3 contacts, each contact providing an orthogonal set consisting of a normal force and 2 resulting frictional forces (for a total of 9 contact wrenches exerted on the object). Designs as simple as 2 single-jointed fingers or 1 multi-jointed finger plus the palm could then completely restrain an object.

\section{Dealing with Friction}

Many different ways of dealing with frictional forces in grasping have been developed [Ohwovoriole 87, Nguyen 86, Salisbury 85, Abel 85]. For our purposes in testing hand designs, we have used essentially the method of Salisbury; we assume the contact forces and coefficients of friction are adequate to cause frictional forces resisting appropriate external forces. This assumption allows us to simplify the stability algorithm since we are not required to deal with coefficients of friction or magnitudes of normal forces. In performing a testing series against a range of possible designs, as long as the test remains the same for each, it will create a relative ranking of grasping ability. We will use this ranking to make recommendations for an optimum end effector configuration. Because of these assumptions, predicted stable 
grasps near the stability limit may be unstable for a real world end effector. Further discussion on this aspect of stability is contained in Chapter 5.

Each contact wrench is assumed to give rise to 2 other mutually orthogonal wrenches representing frictional forces. Since we are not concerned with the magnitudes of frictional forces, contact wrenches are represented by their direction only.

\section{Virtual Work}

Merely providing 7 forces on the object is not enough to guarantee immobilization; relationships between the forces must be investigated. The use of screw and wrench theory in understanding grasp stability was extended by Ohwovoriole and Roth [Ohwovoriole 81]. They defined 3 types of screw pairs: reciprocal, repelling and contrary.

Two screws are called reciprocal screw pairs if the virtual work between a twist and a wrench associated with the two screws is zero. In this case either the contact point does not move or it moves orthogonally to the force. This can be understood by considering two friction point contacts on a sphere. Even with the contact points collinear and intersecting the center, the sphere is free to rotate about the line through the contact points. No work is done on the ball by the contact forces thus the contact wrenches are reciprocal to the twist which the sphere can undergo.

Two screws form a repelling pair if the virtual work between a twist and 
a wrench associated with the screws is greater than zero. Positive work is done by the wrench in accomplishing the twist. In this case the fingers could continue to close, causing motion of the object we are attempting to restrain. Motion away from the contact points is possible, so we do not have complete restraint.

If the virtual work between a twist and a wrench associated with the two screws is negative, the pairs are called contrary screws. The only allowed motion is into the contact points. Since the finger links are (assumed to be) rigid, no motion is possible and the object is completely constrained [Ohwovoriole 81].

To test for constraint with frictionless point contacts, it is only necessary to determine if there are any reciprocal or repelling twists to the set of contact wrenches. This is done by checking for solutions to a system of linear inequalities which represent the virtual coefficients of the known contact wrenches with a general external twist. The solution technique is derived from a paper by Goldman and Tucker [Kuhn 56] and is described concisely in Appendix B of a paper by Ohwovoriole and Roth [Ohwovoriole 81].

Our treatment of wrenches arising from frictional forces requires modification of these techniques. The requirement that no reciprocal or repelling wrenches exist is the case for unidirectional contact wrenches and its use for frictional contacts would be overly restrictive, since frictional wrenches are bi-directional. A method that ignores this could indicate instability even 
with a stable grasp. We define stability as a condition where:

- no twists exist which are reciprocal to the set of resisting wrenches

- no twists exist which are repelling to the wrenches representing normal forces

This ensures that normal forces point in the correct direction to give rise to frictional forces and considers the bi-directional nature of frictional forces.

In contrast, Salisbury's algebraic approach handles the same problem in a different way; it determines if a grasp can resist an arbitrary external wrench. In equation (1), $\boldsymbol{W}$ is the $6 \times \boldsymbol{n}$ matrix of contact wrench directions,

$$
W C=W_{\text {ext }}
$$

c is the $\boldsymbol{n} \times 1$ matrix of contact wrench magnitudes, $\boldsymbol{W}_{\text {ext }}$ is a general external wrench, and $\boldsymbol{n}$ is the number of restraining wrenches. $\boldsymbol{W}$ is arranged so that the first $\mathrm{p}$ elements represent the unidirectional normal wrenches. The corresponding $\mathrm{p}$ elements of $c$ must then be positive to ensure these unidirectional forces are actually pointing in the correct direction. Matrix $\boldsymbol{c}$ can be written as the sum of a particular and a homogeneous matrix, so that: 


$$
c=c_{p}+\lambda c_{h}
$$

The homogeneous solution $\boldsymbol{c}_{\boldsymbol{h}}$ is found by solving equation (1) with the right hand side replaced by 0 . The particular solution is determined by the value of $W_{\text {ext }}$. If we can find a homogeneous solution whose first $\mathrm{p}$ elements are of like sign, then for a given $c_{p}$, we can determine a $\lambda$ so that the first $p$ elements of $c$ are same signed [Lakshminarayana 78]. This means the grasp is stable. The difference between the two methods of determining stability is in the use of the above homogenous solution instead of the sign of virtual work done by the grasp.

\section{Mathematics Used in the Stability Algorithm}

Merely stating the stability conditions belies the difficulty of determining whether they are actually met. To determine this, we must prove or disprove the existence of a solution to a set of linear inequalities. The virtual coefficients are determined by multiplying force components of a wrench by translational components of a generalized twist and torque components of the wrench by rotational components of the generalized twist. In figure 1 , wrench $W 1$ is represented as $[0,1,0,0,0,0]$. The generalized twist is represented as $[T 1, T 2, T 3, T 4, T 5, T 6] ; T 1, T 2$, and $T 3$ are rotations around the $\mathrm{x}, \mathrm{y}$ and $\mathrm{z}$ axes and $\mathbf{T 4}, \boldsymbol{T 5}$ and $\boldsymbol{T} 6$ are translational motions in the $\mathrm{x}, \mathrm{y}$ and $\mathrm{z}$ directions. We form a matrix of virtual coefficients which takes into 
account all contact wrenches. For the condition in figure 1 the set of contact wrenches is:

$$
\begin{aligned}
& \boldsymbol{W} 1=[0,1,0,0,0,0] \\
& \boldsymbol{W} \mathbf{2}=[-1,0,0,0,0,0] \\
& \mathbf{W} 3=[1,0,0,0,0,0] \\
& \boldsymbol{W} 4=[1,0,0,0,0,1] \\
& \mathbf{W 5}=[0,1,0,0,0,1] \\
& \boldsymbol{W} \mathbf{6}=[0,-1,0,0,0,1] \\
& \boldsymbol{W} 7=[0,0,1,-1,0,0] \\
& W 8=[0,0,1,0,-1,0] \\
& \text { W9 }=[0,0,1,0,1,0]
\end{aligned}
$$

The virtual coefficients generated are:

$$
\left|\begin{array}{cccccc}
0 & 0 & 0 & 0 & 1 & 0 \\
0 & 0 & 0 & -1 & 0 & 0 \\
0 & 0 & 0 & 1 & 0 & 0 \\
0 & 0 & 1 & 1 & 0 & 0 \\
0 & 0 & 1 & 0 & 1 & 0 \\
0 & 0 & 1 & 0 & -1 & 0 \\
-1 & 0 & 0 & 0 & 0 & 1 \\
0 & -1 & 0 & 0 & 0 & 1 \\
0 & 1 & 0 & 0 & 0 & 1
\end{array}\right|\left|\begin{array}{l}
T 1 \\
T 2 \\
T 3 \\
T 4 \\
T 5 \\
T 6
\end{array}\right|
$$

We check for reciprocal wrenches to the set of restraining wrenches by determining whether the matrix equation (4) has any solutions, where the left hand side represents the virtual coefficients of the restraining wrenches with a generalized twist. This is equivalent to determining if the null space of equation (4) has any entries. If the null space has non-trivial elements, the 


$$
W T=0
$$

grasp is labelled unstable.

If the null space is empty, we then consider the normal wrenches alone. To check for wrenches repelling to the normal wrenches, we must see if

$$
W_{n} T>0
$$

solutions exist to relationship (5), where the left hand side represents the virtual coefficients of the normal wrenches with a generalized twist. This is done using the following theorem from Gale: "Exactly one of the following alternatives holds. Either the equation

$$
x A=0
$$

has a semipositive solution or the inequality

$$
\text { A } y>0
$$

has a solution."[Gale 60].

Gale defines a vector $\mathrm{x}$ to be semipositive if its elements are all greater than or equal to zero but not all equal to zero. Using equality (6) we can check for solutions to relationship (7). Taking the transpose of equation (6) we have:

$$
A^{t} x^{t}=0
$$

Matrix $\boldsymbol{A}$ is made up only of the normal wrenches. We determine an 
orthonormal basis for the null set of equation (8) and check if any of these basis vectors are semipositive. If so, we have met the first condition of the above theorem and know that no repelling wrenches exist. Even if none of the basis vectors are semipositive, however, it may still be possible to form a linear combination of these basis vectors that will be semipositive. If $\boldsymbol{X}_{1}, \boldsymbol{X}_{2}, \ldots, \boldsymbol{X}_{n}$ are the basis vectors, then we may represent this problem as:

$$
X_{1} \lambda_{1}+X_{2} \lambda_{2}+\cdots+X_{n} \lambda_{n} \geq 0
$$

or

$$
\left|\begin{array}{llll}
X_{1} & X_{2} & \cdots & X_{n}
\end{array}\right|\left|\begin{array}{c}
\lambda_{1} \\
\lambda_{2} \\
\vdots \\
\lambda_{n}
\end{array}\right| \geq 0
$$

We must determine if any solutions exist to the relationship (10).

Our method for doing this is adapted from Goldman and Tucker [Kuhn 56]. We form a matrix whose columns are $\boldsymbol{X}_{1}, \boldsymbol{X}_{2}, \ldots, \boldsymbol{X}_{n}$ and choose (in all possible ways) a subset of $n-1$ linearly independent rows of this matrix. Call this sub-matrix $N$. We determine all non-trivial solutions to:

$$
N Z=0
$$

From the remaining rows not chosen for $N$, we form the matrix $M$. For each solution $\boldsymbol{Z}_{\boldsymbol{p}}$ to equation (11), we evaluate the product $\boldsymbol{M}$ times $\boldsymbol{Z}_{\boldsymbol{p}}$ and $\boldsymbol{M}$ times $-Z_{p}$. If either of the following are true: 


$$
\begin{gathered}
M Z_{p} \geq 0 \\
M\left(-Z_{p}\right) \geq 0
\end{gathered}
$$

then we have found a semipositive solution to equation (6). By Gale's theorem, this means no solution to relationship (7) can exist and there are no repelling wrenches to the normal wrenches. Thus this grasp is stable. If no semipositive solution to equation (6) is found, relationship (7) must have a solution, meaning that repelling wrenches to the normal wrenches do exist and the grasp is unstable.

The procedure deals with the existence of solutions instead of actual determination of solutions, so it is unable to find an example of an unresisted twist. For this reason, the twist discussed in example 2 below was determined by inspection.

The portion of this method which determines the existence of solutions to equation (9) is very similar to the technique for finding solutions to $\mathrm{AX}<0$ explained in Appendix B of Ohwovoriole and Roth. It differs in several respects: it finds solutions to $\boldsymbol{A X} \geq \mathbf{0}$ instead of $\boldsymbol{A X} \leq \boldsymbol{0}$, solutions with all zero elements are unacceptable, and the checks involving the $M$ matrix (relationships 12 and 13) use $\geq$ instead of $>$.

\section{Conceptual Examples of Stability Determination}

\section{Example 1:}


A cylinder is grasped by a simple two finger end effector as pictured in Figure 1. The set of contact wrenches generated is:

$$
\begin{aligned}
& \text { W1 }=[0,1,0,0,0,0] \\
& \text { W2 }=[-1,0,0,0,0,0] \\
& \text { W3 }=[1,0,0,0,0,0] \\
& \text { W4 }=[1,0,0,0,0,1] \\
& \text { W5 }=[0,1,0,0,0,1] \\
& \text { W6 }=[0,-1,0,0,0,1] \\
& \text { W7 }=[0,0,1,-1,0,0] \\
& \text { W8 }=[0,0,1,0,-1,0] \\
& \text { W9 }=[0,0,1,0,1,0]
\end{aligned}
$$

The virtual coefficients generated are:

$$
\left|\begin{array}{cccccc}
0 & 0 & 0 & 0 & 1 & 0 \\
0 & 0 & 0 & -1 & 0 & 0 \\
0 & 0 & 0 & 1 & 0 & 0 \\
0 & 0 & 1 & 1 & 0 & 0 \\
0 & 0 & 1 & 0 & 1 & 0 \\
0 & 0 & 1 & 0 & -1 & 0 \\
-1 & 0 & 0 & 0 & 0 & 1 \\
0 & -1 & 0 & 0 & 0 & 1 \\
0 & 1 & 0 & 0 & 0 & 1
\end{array}\right|\left|\begin{array}{c}
T 1 \\
T 2 \\
T 3 \\
T 4 \\
T 5 \\
T 6
\end{array}\right|
$$

We need to determine if solutions exist to the relationships (15) and (16), where

$$
\left.\begin{array}{cccccc}
A T=0 \\
0 & 0 & 0 & 0 & 1 & 0 \\
0 & 0 & 0 & -1 & 0 & 0 \\
0 & 0 & 0 & 1 & 0 & 0
\end{array}\right|_{T>0}
$$


$\boldsymbol{A}$ is the matrix associated with virtual coefficients and $\boldsymbol{T}$ represents a generalized twist. We find that there are no solutions to either of these relationships, therefore neither reciprocal wrenches nor wrenches repelling to the friction-causing normal wrenches exist. We thus predict this grasp is stable.

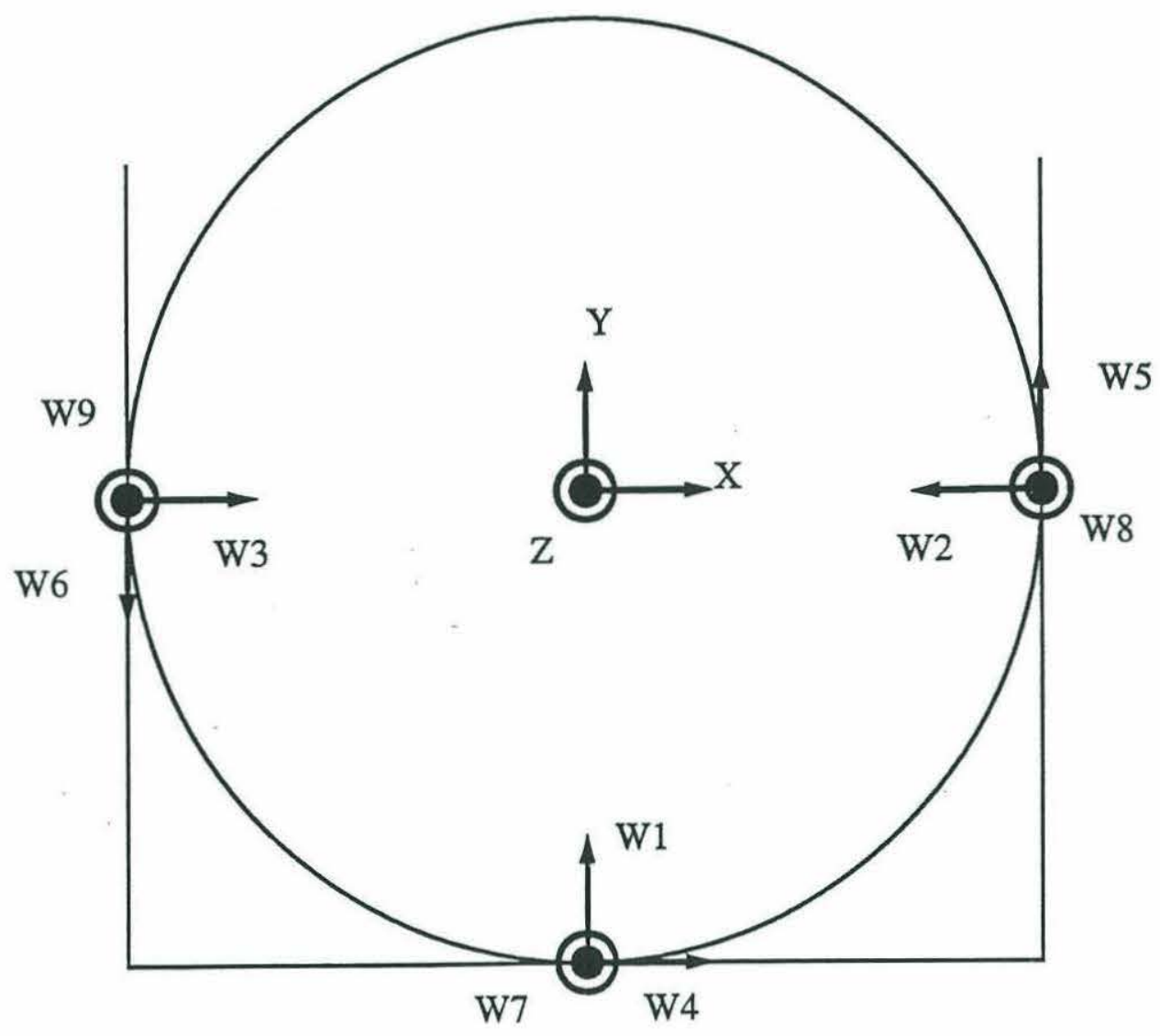

- Denotes Arrow Pointing Out of Page

Figure 1: Example 1 


\section{Example 2:}

A cylinder is lying on a flat surface against a vertical finger as pictured in figure 2. We see the cylinder is unrestrained against forces that tend to roll it away from the finger or up the finger. The contact wrenches are:

$$
\begin{aligned}
& \text { W1 }=[-1,0,0,0,0,0] \\
& \text { W2 }=[0,1,0,-1,0,0] \\
& \text { W3 }=[0,1,0,1,0,0] \\
& \text { W4 }=[1,0,0,0,1,1] \\
& \text { W5 }=[0,0,1,-1,0,0] \\
& \text { W6 }=[1,0,0,0,-1,1] \\
& \text { W7 }=[0,0,1,-1,0,0] \\
& \text { W8 }=[0,1,0,0,0,1] \\
& \text { W9 }=[0,0,1,0,-1,0]
\end{aligned}
$$

and the virtual coefficients are depicted in (17). We find there are no twists

$$
\left|\begin{array}{cccccc}
0 & 0 & 0 & -1 & 0 & 0 \\
-1 & 0 & 0 & 0 & 1 & 0 \\
1 & 0 & 0 & 0 & 1 & 0 \\
0 & 1 & 1 & 1 & 0 & 0 \\
-1 & 0 & 0 & 0 & 0 & 1 \\
0 & -1 & 1 & 1 & 0 & 0 \\
-1 & 0 & 0 & 0 & 0 & 1 \\
0 & 0 & 1 & 0 & 1 & 0 \\
0 & -1 & 0 & 0 & 0 & 1
\end{array}\right| \begin{aligned}
& T 1 \\
& T 2 \\
& T 3 \\
& T 4 \\
& T 5 \\
& T 6
\end{aligned} \mid
$$

reciprocal to the set of contact wrenches since the null set of: 


$$
\left|\begin{array}{cccccc}
0 & 0 & 0 & -1 & 0 & 0 \\
-1 & 0 & 0 & 0 & 1 & 0 \\
1 & 0 & 0 & 0 & 1 & 0 \\
0 & 1 & 1 & 1 & 0 & 0 \\
-1 & 0 & 0 & 0 & 0 & 1 \\
0 & -1 & 1 & 1 & 0 & 0 \\
-1 & 0 & 0 & 0 & 0 & 1 \\
0 & 0 & 1 & 0 & 1 & 0 \\
0 & -1 & 0 & 0 & 0 & 1
\end{array}\right|\left|\begin{array}{l}
T 1 \\
T 2 \\
T 3 \\
T 4 \\
T 5 \\
T 6
\end{array}\right|=0
$$

is empty. There is a solution to relationship (19), however.

$$
\left|\begin{array}{cccccc}
0 & 0 & 0 & -1 & 0 & 0 \\
-1 & 0 & 0 & 0 & 1 & 0 \\
1 & 0 & 0 & 0 & 1 & 0
\end{array}\right|
$$

One of the many twists that cannot be resisted by this grasp is $[0,0,0,-1,1,0]$. This corresponds to a translation in the - $\mathrm{x}$ direction combined with an equal translation in the $+y$ direction. Since there is at least one wrench that can not be resisted, the grasp is unstable.

\section{Characterization of Stability Criteria: Form or Force Closure?}

One might ask whether this way of determining grasp stability is a form or a force closure method. First, since the consideration of frictional forces is 


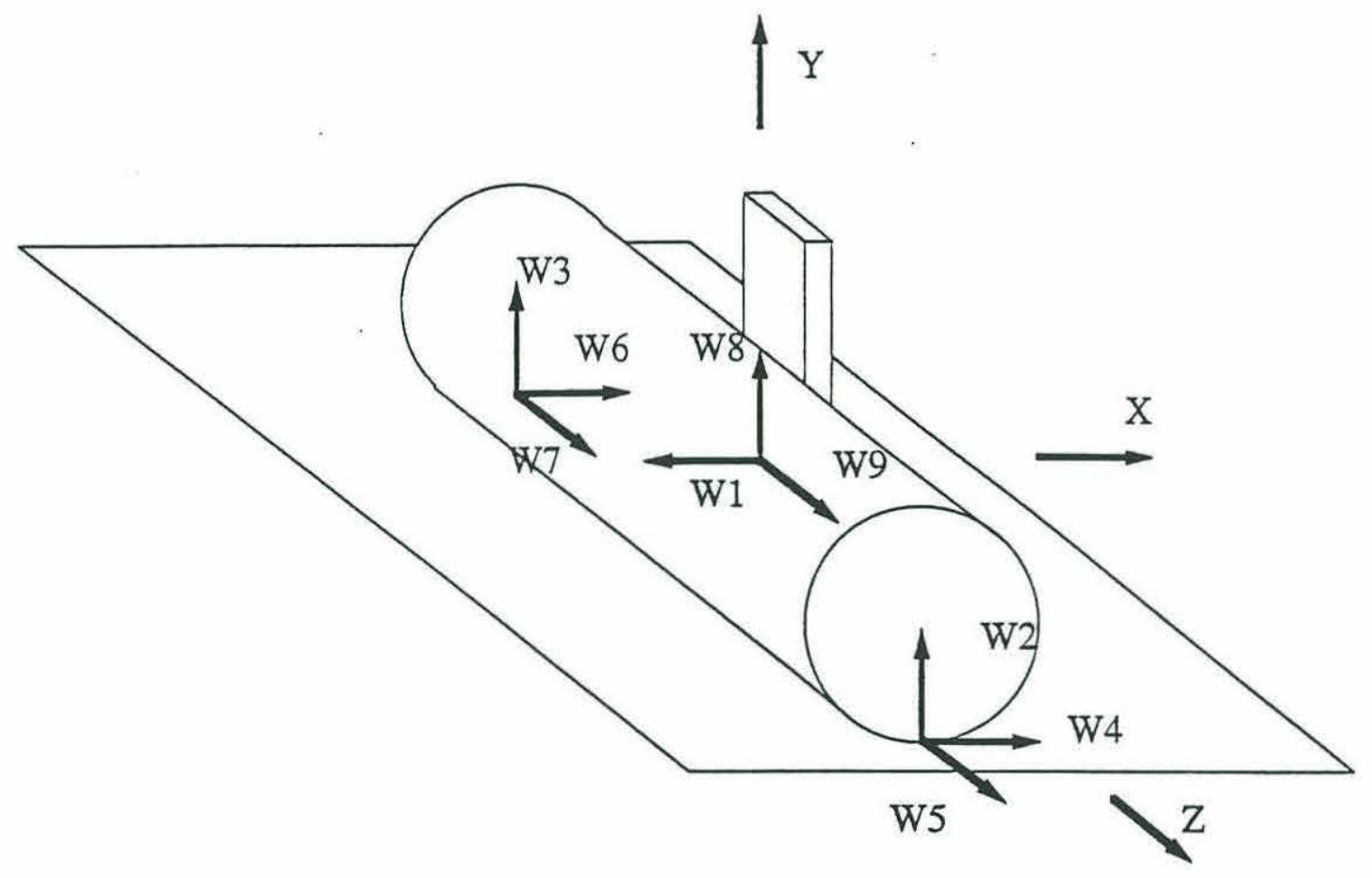

Figure 2: Example 2

central to the method, it is closer to force closure than to form closure. However, a force closure grasp can be judged stable due to the presence of an external force. Ulrich uses a clothes hanger on a hanger rod as an example of force closure [Ulrich 88]. In this example, the situation is stable due to the presence of an external wrench (gravitational force). A simulation of underwater grasping dependent on an external force for stability determination would be useless, since grasped objects can either be positively, negatively or neutrally buoyant. The algorithm is best characterized as a force closure test 
which considers only contact and frictional forces. 


\section{Chapter 3}

\section{The Grasp Tester}

\section{Background on Grasp Tester}

The purpose of this work is the evaluation of end effector designs to choose the best for our purposes. In order to do this, we must develop a grasp simulation tester using representative objects to evaluate candidate hand designs. Selection of appropriate test objects is important and will be discussed below. We must also standardize the assumptions about hand configurations to provide a baseline for evaluation.

The simulation program was written in Matlab since numerous matrix manipulations were required and Matlab provides excellent tools for this work. The methods for determining grasp stability were outlined in Chapter 2. This chapter deals with the simulations that provide the contact wrenches for evaluation by the stability algorithm.

\section{Hand Assumptions for the Grasp Tester}

Test standardization and the requirement for fair testing of all designs required a standard set of assumptions about the end effectors under consideration. We arrived at the following assumptions based on previous 
works in the field and a knowledge of basic techniques for simplification of hand design.

A circular palm with fingers located on the periphery was assumed. The program evaluated designs with from 1 to 4 fingers and 1 to 3 links per finger. All fingers were assumed to "point" towards the center of the palm, meaning that the base joint axis was tangent to the palm circumference for all fingers. Fingers were assumed to be equally spaced about the palm and all fingers had the same length and number of links. Finger joints incorporate a means of compliant coupling as discussed by Ulrich [Ulrich 88]. Finger motion is thus described as follows:

- The first joint closes until contact occurs

- The second joint then closes until contact occurs

- The third joint closes until contact occurs

Collisions between fingers were ignored. Fingers were not allowed to bend backwards as illustrated in figure 3 . If the first link was too short to make contact, the simulation considered the possibility that initial contact could occur on a subsequent link. In the simulation, the fingers have no width and are treated as lines only. This precludes use of the simulation for study of finger or palm shape variations; their influence on grasp stability is a topic for further study. 


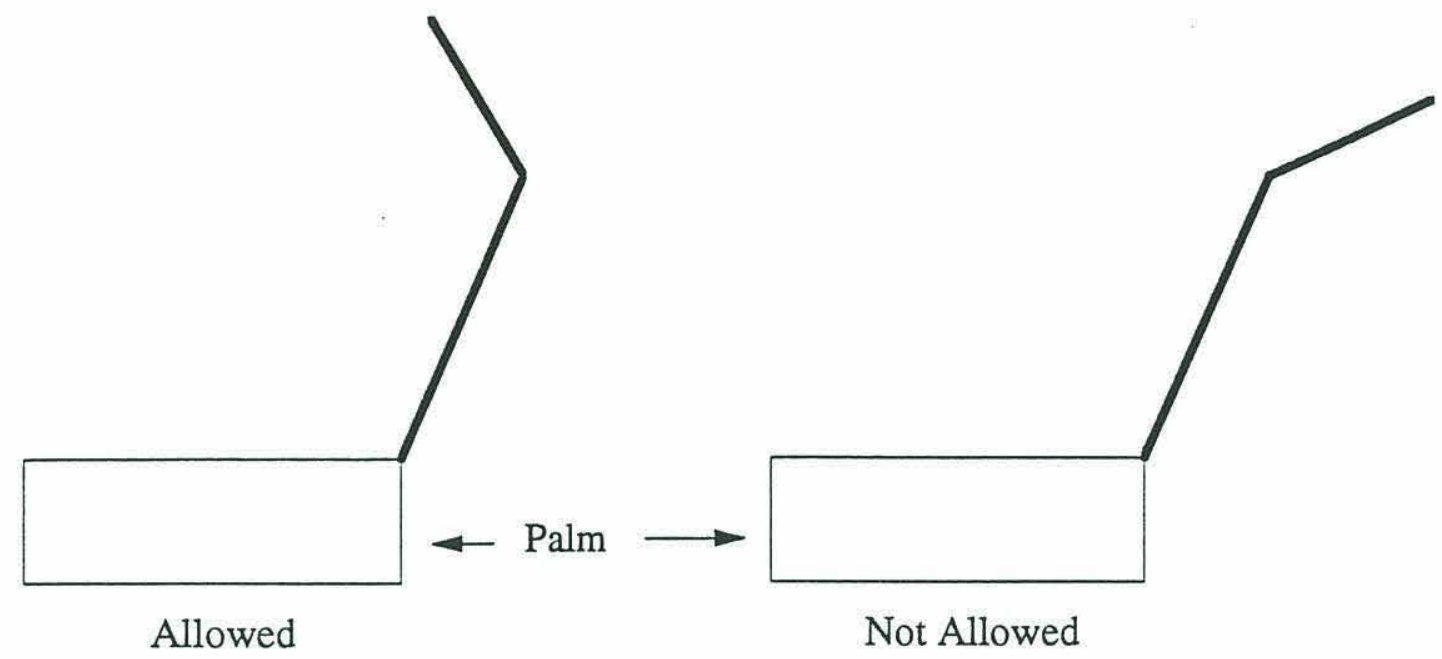

Figure 3: Allowed and disallowed finger positions

\section{Object Selection}

Our goals require that we conduct grasp testing with objects that would relate to the deep sea research mission of the Jason/Medea vehicle system (Figure 4). Jason is an advanced Remotely Operated Vehicle (ROV) developed by Woods Hole Oceanographic Institution (WHOI). Medea is an unpowered towed sled which is used both to decouple wave motion from Jason and to provide a wide area survey capability. Several experienced ROV/Deep Submergence Vehicle (DSV) pilots were interviewed to obtain their assessment of typical mission requirements. Using this information along with the guidance of others with experience in deep sea manipulation, we selected three representative test shapes. A sphere was chosen to represent various 


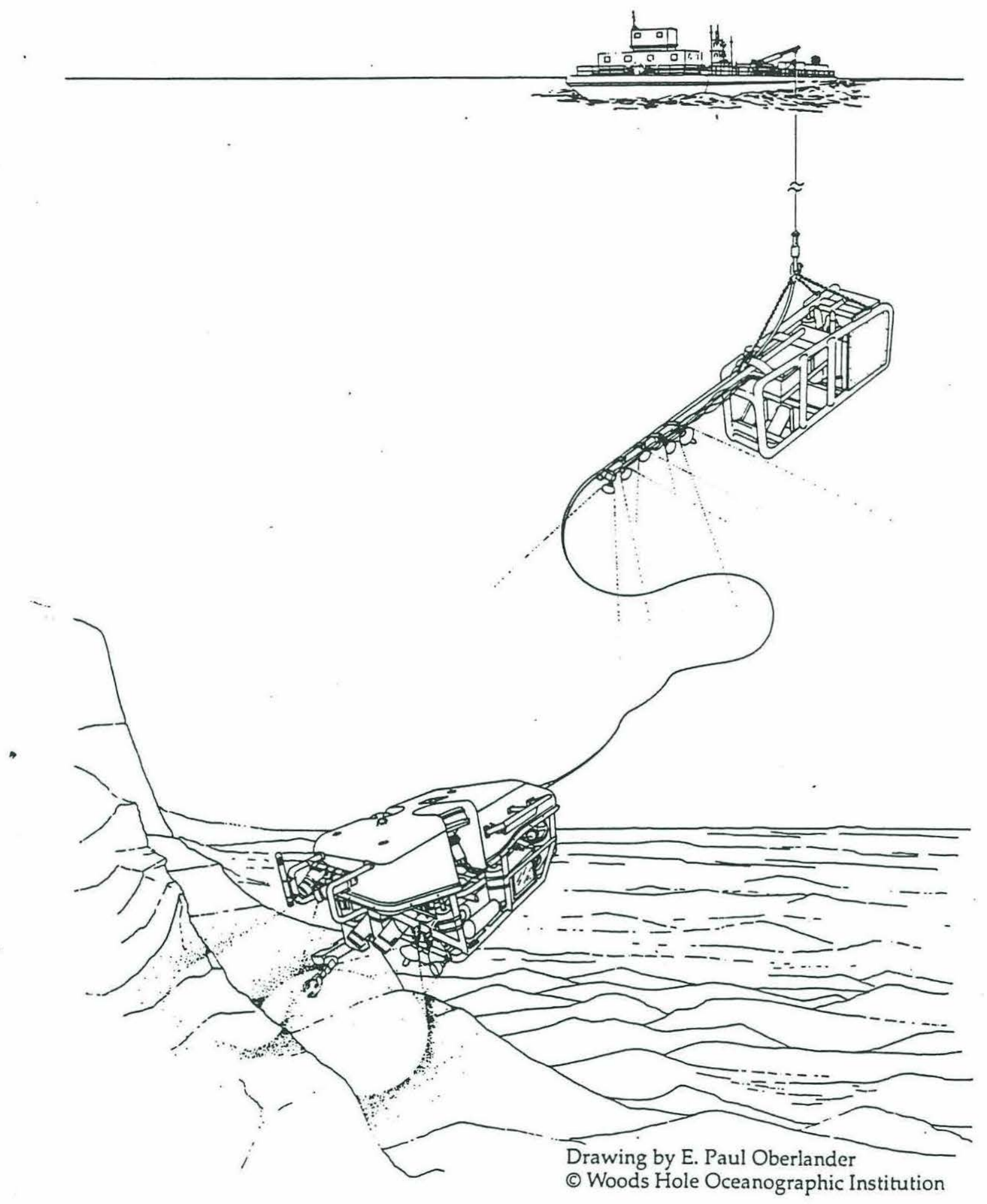

Figure 4: The Jason/Medea vehicle system 
biological and geological samples which typically approximate a spheroid, such as shells, small animals, and rocks. A sphere is an adequate representation of these objects because its symmetry makes it more difficult to stably grasp than other spheroids. The second test shape is a cube. It was chosen to represent manmade objects which are frequently rectangular in shape. This test simulates grasping tasks required on an object recovery mission. The final shape is a cylinder of infinite length. This was chosen because many hydrographic instruments are cylindrically shaped, such as core tubes, and must be deployed manually.

\section{The Sphere Test Simulation}

The sphere test simulation is the easiest of the three to implement. The basic concept of each of the simulations is to reduce the three dimensional intersection problem to two dimensions in the plane of the respective finger. For each finger, the sphere tester determines the size and location of the two dimensional circle of intersection of the finger plane and the sphere. It then solves the two dimensional intersection problem for each finger link and converts to the corresponding three dimensional intersection point. If the finger links are not long enough to reach the calculated intersection point, this point is used as the initial value for a numerical routine which determines a solution the finger can reach. The intersections resulting from this routine are referred to as interior intersections. For the second and third links, the 
program checks for contact with the palm, as would happen if multi-jointed fingers were wrapped around a very small object. If finger contact with the palm is predicted, that link's intersection is ignored. Finally, the program calculates one intersection for the single contact between the sphere and the palm.

For each intersection developed, the simulation generates a normal wrench which is orthogonal to the surface of the object. It then uses a vector cross product with the finger link direction to give a vector orthogonal to the previous two. A second vector cross product between the normal and the first orthogonal wrench provides a second orthogonal vector. The two non-normal vectors along with the location of the intersection and center points of the circle are used to generate the wrenches representing frictional forces. This method is used for all contacts in the sphere test routine, although it is actually only required for interior intersections. For tangent intersections on a sphere, the finger link direction is orthogonal to the normal vector and the above method could be simplified to one cross product. The more general method is used in the simulation to simplify program structure.

The set of contact wrenches generated are passed to the stability module, which returns the determination stable or unstable. The sphere test routine accepts inputs of finger number, number of links per finger, length of links, radius of sphere, radius of palm, and location of sphere in palm. No motion of the sphere is considered during the grasping process; if a grasp 
results which would either allow or cause motion, the grasp is assessed to be unstable. Modification of these methods to consider motions during grasping is a topic for further development.

\section{The Cylinder Tester}

Like the sphere tester, the cylinder tester solves the three dimensional grasping problem by resolving it into a sequence of two dimensional problems. Unlike the sphere, however, the two dimensional intersections of the cylinder and the finger plane are nearly always elliptical. The simulation uses an analytical expression to determine the tangent to the ellipse through the point defined by the respective joint location. It can deal with objects of elliptical vice circular cross sections, although all test runs for this work were done using a cylinder. Once a prospective intersection is determined, the program checks for adequate finger length, and if inadequate, checks the length of the rest of the finger to see if intersection is possible at all. If the finger is long enough to intersect the ellipse but cannot reach the tangential intersection point, a finger tip intersection is calculated using a numerical routine (an interior intersection).

After determining the intersection point, the simulation calculates a normal using the tangent at that point, and develops normal and frictional wrenches in a similar fashion to the sphere above. The simulation then checks the second and third joints, if they exist, for palm contact and discards that 
link's intersection if palm contact is predicted.

2 wrenches are then calculated to represent the cylinder's contact with the palm. For this, the simulation uses the endpoints of the line of intersection between the cylinder's surface and the palm. According to Nguyen, line contact can be adequately represented by 2 wrenches, one at each end of the line [Nguyen 87]. The program generates orthogonal sets of contact wrenches at these points as described above. It assumes an infinitely long cylinder and accepts inputs of major and minor axes (for the cylinder), left/right position, and orientation of the cylinder in the hand. The simulation also accepts similar finger inputs to those described under the sphere test.

\section{The Cube Tester}

The cube simulation posed a different problem than the sphere or cylinder tester. For the previous objects, the 2 dimensional intersections were cylindrical or elliptical. The cube tester intersections were rectangular and the function describing the shape is generally non-differentiable, requiring a different approach to that used previously.

In the simulation, the cube is described using methods from the field of computer graphics. A matrix is formed from its vertices and a second matrix (called the connections matrix) encodes vertices which are connected. For each finger and each link, a check is made to determine the closest vertex. In many cases, 2 vertices are equal distances from the link. This simulation picks one 
and uses it for the basis of further calculations. The results are invariant of which closest vertex is chosen. Once the closest vertex has been determined, the three edges connecting it to other vertices are examined to see if they could intersect the finger. The edges are defined not only by their position and direction, but also by their length, so this check comes up with at most one possible intersection point between the finger and an edge of the cube. The link and if necessary the entire finger are checked for adequate length. If length is inadequate, the program calculates an interior intersection using trigonometric and geometric arguments. The simulation considers the possibility of finger contact with the palm for the second and third links, and if predicted, that link's intersection is discarded.

Once the intersection point has been determined, the program calculates the inward normal and the orthogonal frictional wrench directions. If the point is the result of an interior intersection, obtaining the direction of the inward normal is straightforward. For edge intersections, however, the direction of the inward normal is assumed to be a direction perpendicular to both the edge and the finger. The finger direction is then used for one of the frictional force directions and the vector cross product of the normal direction with the finger direction gives the second friction wrench direction.

If the position of the cube in the palm is such that one of the base finger joints is covered by the cube, the grasp is determined to be unstable. Otherwise, the contact wrenches are passed to the stability routine which 
returns the determination of stable or unstable. The cube simulation accepts the same finger inputs as the two other modules in addition to the length of a side of the cube, position of the cube in the palm and rotary orientation of the cube.

\section{Scoring and Evaluation}

A master grasp testing routine combines the above programs to successively iterate through combinations of one to four fingers and one to three links per finger. Lengths in the program were normalized by dividing by palm radius. Effectively, palm radius was set to one and finger and object size were referenced to this length. The effect of varying finger length was removed by assigning a set finger length, 1.5 times total palm width, and holding it constant regardless of number of joints per finger. If this were not done, the effect of using longer fingers would be impossible to distinguish from a variation in number of finger links. Link proportions chosen for testing are discussed in the chapter on grasp tester results.

For each configuration, the master grasp testing program evaluated the maximum and minimum size objects which could be stably grasped. The raw size score was calculated using the following relationship:

$$
\text { Rawsizescore }=\text { Largestsize }+\frac{1}{10 \times \text { Smallestsize }}
$$

The minimum size test did not differentiate well between configurations; most 
multifingered configurations are capable of stable grasping of each test object down to the smallest tested size. Because of this, the scoring function placed little weight on the results of the small size test.

Also for each configuration, the program determined the maximum amount of mispositioning in the palm for which the object is stable. It did this in the sphere and cube modules by incrementing the object diagonally outward from the palm center and evaluating stability for each increment. For the cylinder determination, the program incremented the cylinder across the palm perpendicular to the long axis of the cylinder. This difference is due to the nature of the object; the infinite length cylinder appears the same whether mispositioned diagonally or just along one axis. The raw position score is the largest positioning increment for which the grasp is stable. Since the size of the object effects stability, this test was done for a standard object size. The sizes used for this test were the following: sphere - unit radius, cylinder - unit length major and minor axes, cube - unit length for a single side. The raw scores for the three objects were added together to obtain a total score for each hand configuration.

A normalized score was calculated to remove bias due to differing scales of raw scores. The scoring routine divided raw scores by a factor making the maximum score for each test one and added results together to give a normalized total score. The raw (unnormalized) total score was useful for comparing various link proportions. The set of link proportions with the 
highest total score was deemed to be the most favorable set. The normalized score was useful in deciding the best combination of fingers and links to maximize grasping ability with the least increase in mechanical complexity. This decision was not based strictly on the highest total score, but was determined by weighing the mechanical complexity versus the normalized grasping score. The results showed an overall similar shape; a rapid rise in score with increased complexity up to a point, then a smaller gain as complexity was further increased. Further results of the grasp tester are covered in Chapter 4. 


\section{Chapter 4}

\section{Design Features and Results}

In previous chapters we have discussed the motivation for this work, the workings of the grasp tester and the stability algorithm. This chapter discusses results and observations of the simulation programs as well as design features which affected our assumptions for the simulation. Results of the grasp tester are included as Appendix 1.

\section{Compliant Coupling}

The simulation assumes compliant coupling between finger joints. This benefits us in two ways; it reduces our dependence on computerized grasp planning and decreases the number of required actuators. As shown by Ulrich, compliant coupling allows design of fingers that automatically wrap around objects, helping to achieve a stable grasp. This automatic wrap around feature eliminates the need for computerized grasp planning algorithms. Grasping can be as simple as extending the hand until palm contact occurs, then closing the fingers until contact has occurred on all joints [Ulrich 88]. Additionally, compliant coupling can reduce the number of actuators required from as many as one per link down to one per finger. Given an simple and reliable method 
of compliant coupling, the reduction in actuators can significantly decrease the complexity of the system and thus provide improved reliability for the end effector.

\section{Palm Prehension}

The use of palm prehension as a design feature also results in improved grasping. For any object, palm contact adds at least 3 contact wrenches (considering frictional point contact). Achieving these contact wrenches with fingers would require at least an additional link, increasing complexity. A palm is required for actuator mounting and to provide a frame for attachment between end effector and manipulator; it should also provide a prehension feature in addition to mechanical support. The inclusion of palm prehension in the design involves no additional links and only slightly increased cost.

\section{Finger Length and Proportion}

Longer total finger length tends to allow grasping of larger objects. To avoid skewing grasp tester results, total finger length was held constant at 1.5 times palm diameter. Palm diameter was fixed at 2 units, so that total length for all finger configurations was 3 units. Finger link proportions were varied in 3 different arrangements. The first set of proportions consisted of equal length finger links, so that with 1 link, the length was 3 units, for 2 links, each was 1.5 units long and with 3 links, each was 1 unit long. The next set was 
made up of links with the next outer joint half as long as the previous. For 2 links, lengths were 2 and 1 units and for 3 links, the lengths were 1.71, .86 and .43 units. The final set approximated anthropomorphic dimensioning. Several human hands were measured, their lengths were normalized and the proportions were averaged. The three link proportions were derived from measurements of the first and middle fingers and the two link proportions were taken from those of the last two links of the thumb. The proportions used were 1.65 and 1.35 for the 2 link tests and $1.425, .9$ and .675 for the 3 link tests. The sets of proportions were referred as the equal length, half length and anthropomorphic proportions. No effort was made to study variations in grasp ability due to finger length.

\section{Comparison of Normalized Results}

The normalized results are useful in the determination of the best finger/link combination to be used for a given set of link proportions. Raw (unnormalized) scores are used in comparisons between different sets of link proportions since the normalization factors differ between these tests. Each set of normalized results can be divided into high and low score areas. The low score area is made up of the single finger/any number of links and the single link/any number of fingers configurations. The high score area is made up of the configurations with from 2 to 4 fingers and from 2 to 3 links. This result is independent of link proportions. We can see that grasp scores increase 


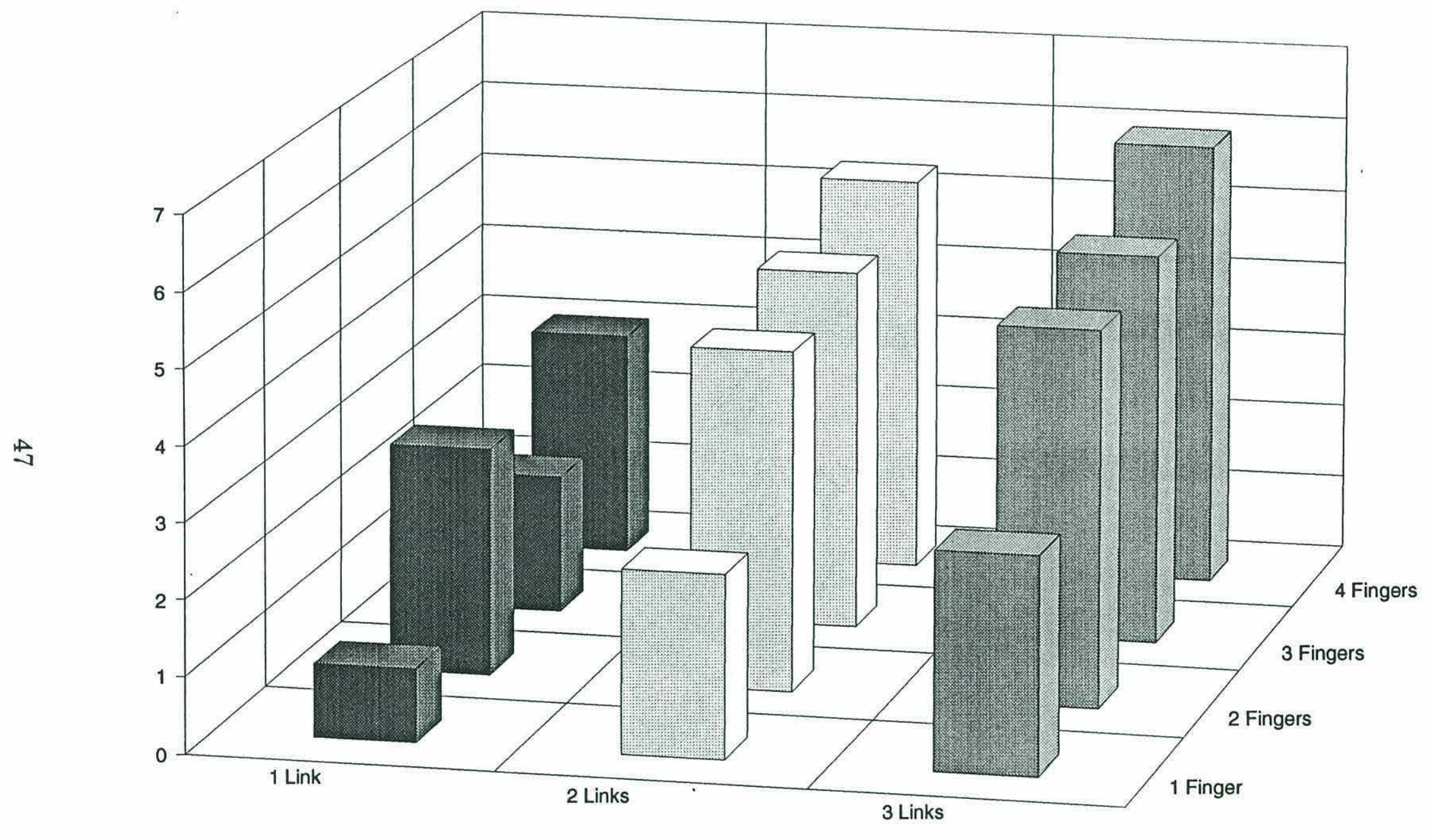

Figure 5: Normalized results for equal proportions 


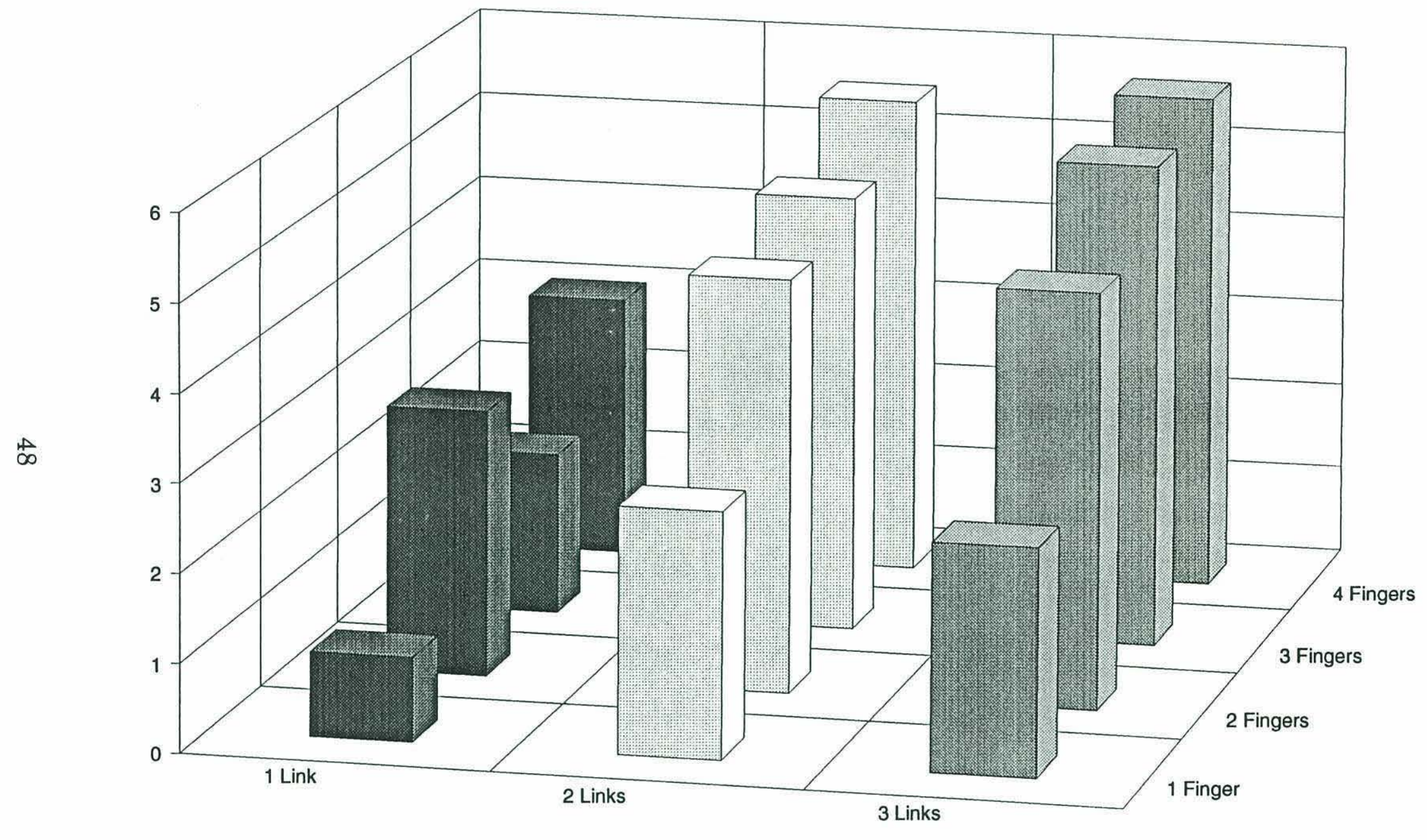

Figure 6: Normalized results for half length proportions 


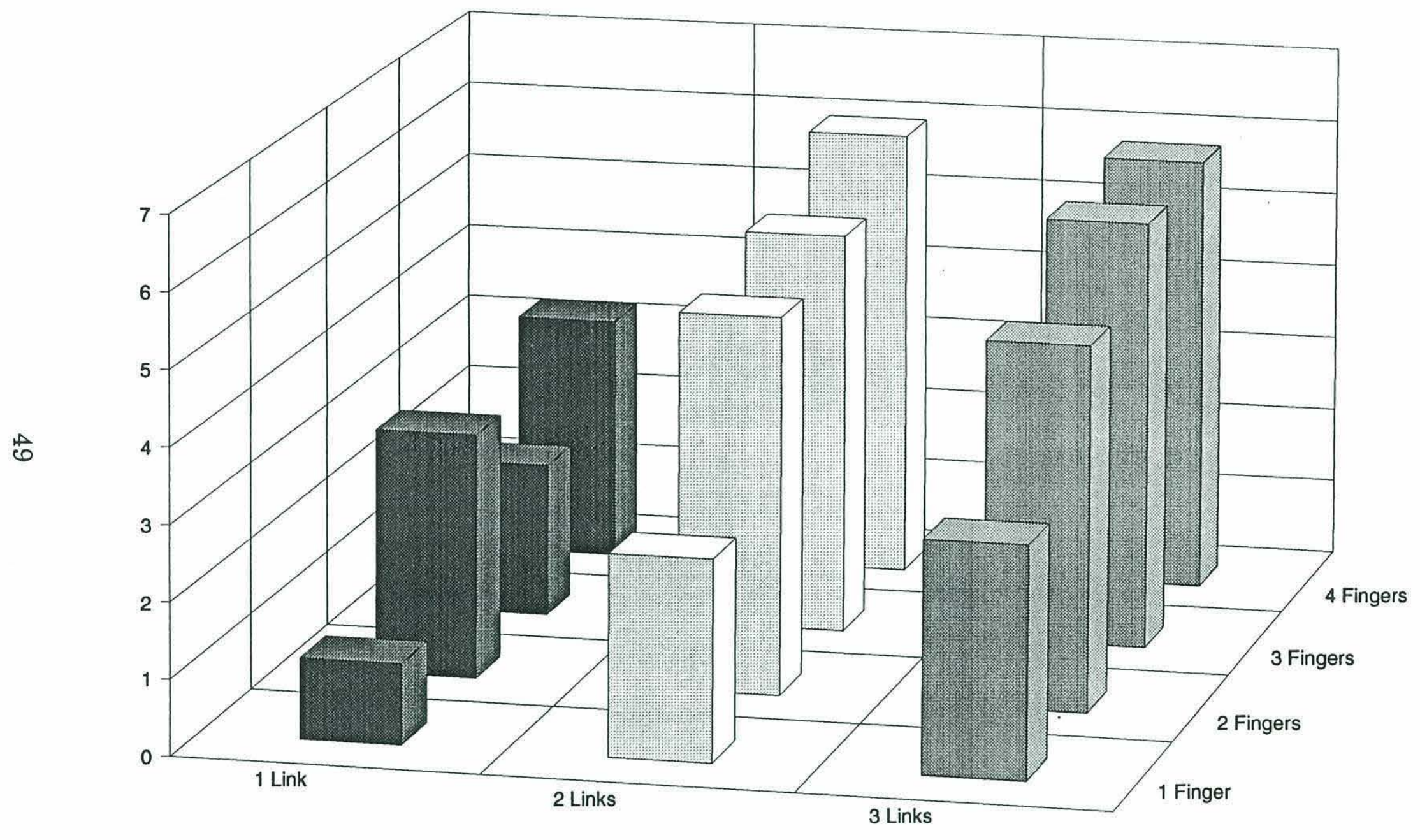

Figure 7: Normalized results for anthropomorphic proportions 
rapidly when going from either any one finger or one link configuration to the two finger/two link configuration. The increase in scores when increasing apparent degrees-of-freedom above this is proportionally much smaller. The choice resulting in the highest grasp score for the simplest design is the two finger, two link end effector.

\section{Finger Proportions}

The raw (un-normalized) total scores are useful for finger proportion comparisons. Considering only the $2 / 2$ configuration, the highest score of 9.4 was obtained for the half length proportions. The other two sets of proportions scored 8.3 (equal length) and 8.7 (anthropomorphic).

Reviewing other configurations, the highest overall score was obtained with the equal length proportions and the $4 / 3$ finger configuration. The largest score for the other two sets of proportions occurred for the $4 / 2$ configuration. The difference in score for the $4 / 3$ configuration was due to an anomaly discussed in the next chapter (see also figure 11). Based on these scores, it would be hard to justify the increased mechanical complexity of four 3 link fingers over ones with 2 links. This can be extended to all cases of increase from 2 to 3 links per finger. In each the change in the normalized score was small and it often decreased, indicating that for our purposes 2 links per finger appears to be optimum.

In the one finger case, both raw and normalized scores show a unique 


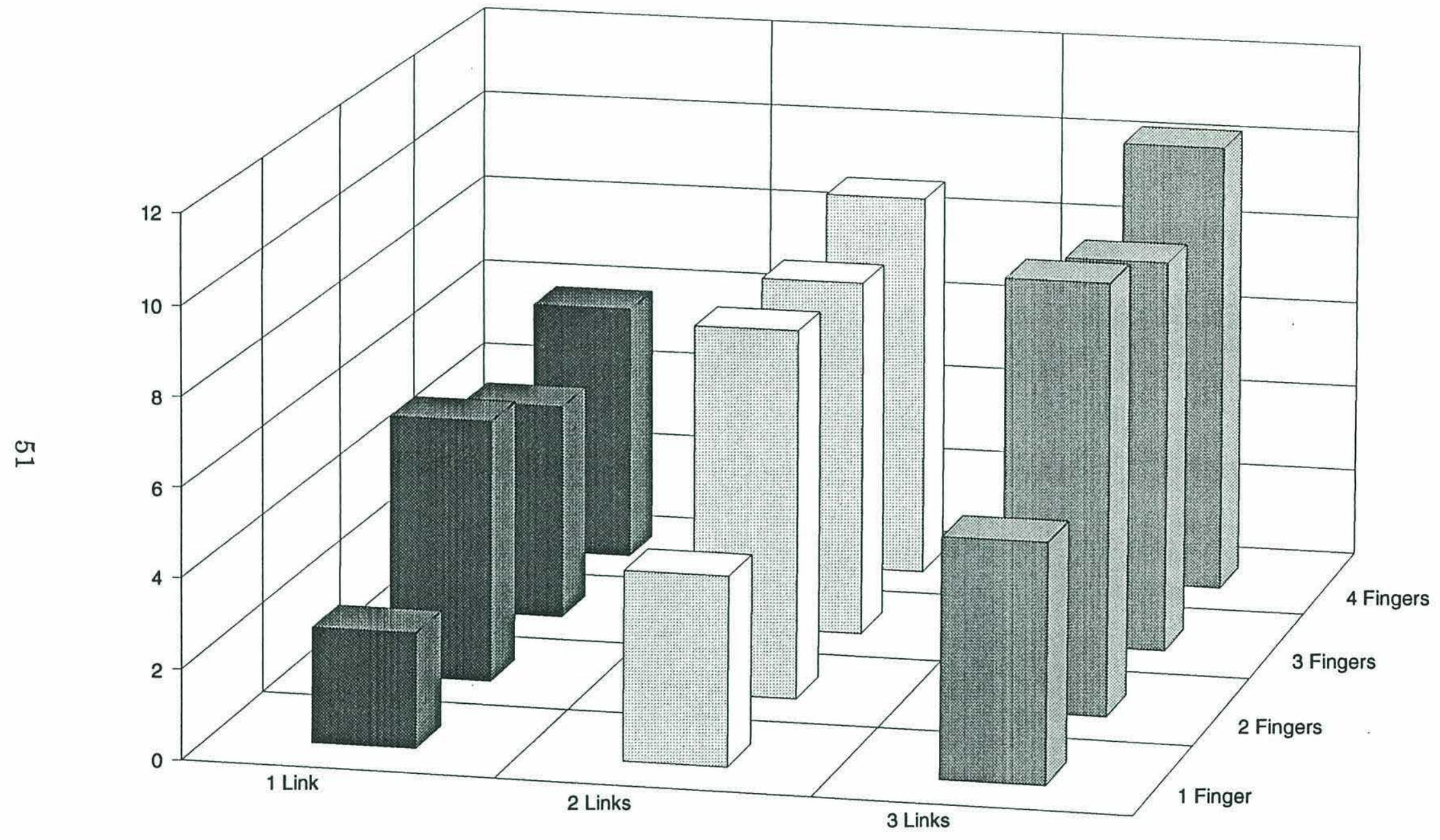

Figure 8: Total scores for equal length proportions 


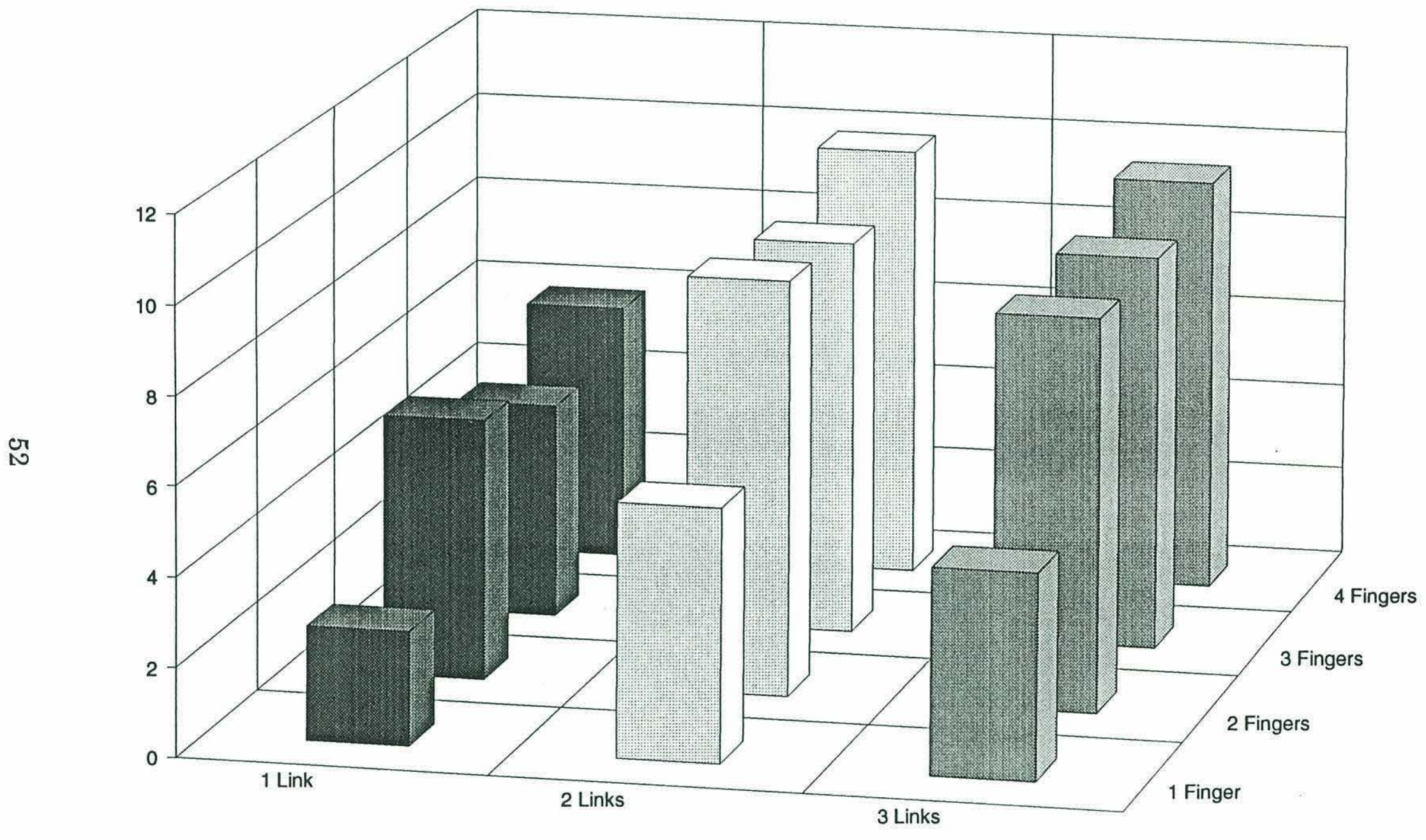

Figure 9: Total scores for half length proportions 


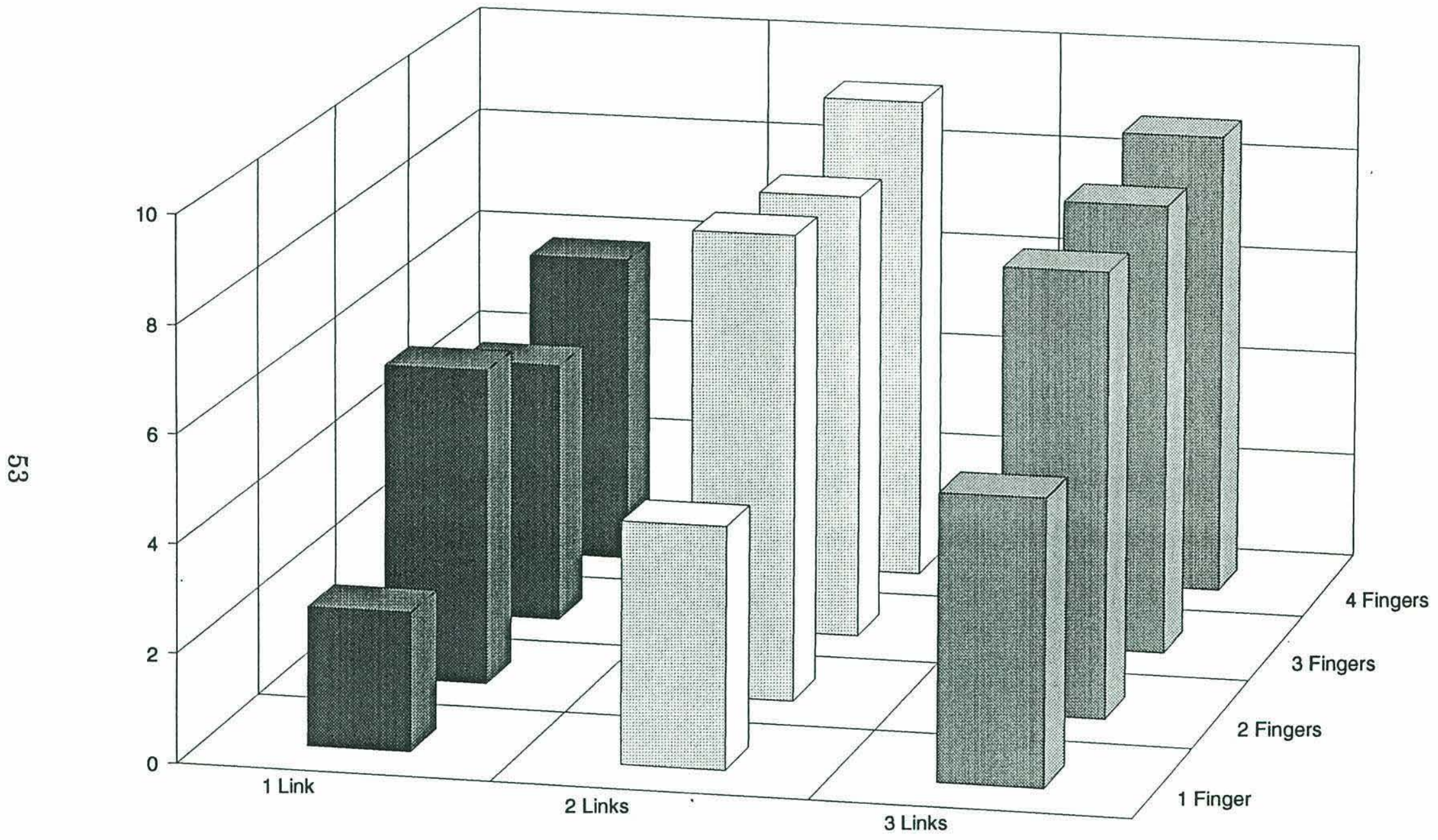

Figure 10: Total scores for anthropomorphic proportions 
trend. For the half length set of proportions, the scores were higher for 2 links than for 3. This is opposite the trend for the other two sets of proportions. Additionally the raw scores for the $1 / 2$ configuration of half length proportions were significantly higher than any other single finger score. This is probably because of the long base joint of this proportion. The link length of 2 units for the base joint is the longest one tested, and affords better wrapping ability since it moves the second link joint farther out from the palm.

\section{Discussion of Individual Tests}

No one test was able to by itself differentiate between the various proportions and configurations. Although we see a generally increasing trend of increased scores with increased link numbers as intuitively expected, some characteristics deserve special attention. The following discussions summarize salient features of individual test results.

\section{Sphere Tests}

The sphere size tests generally show increasing scores as the number of links per finger are increased from 1 to 2 . This is as expected, since an increasing number of links allows improved wrap around abilities, especially as sphere diameter increases above palm diameter. A further increase in the number of links reduced the score in all cases. Increasing the number of fingers improved scores when finger number was increased from 1 to 2 , but 
further increases showed no improvement. This is due to the spherical symmetry of the object. With 2 fingers, adequate contact wrenches were generated for stability; stability is not improved by adding more (for the size test).

In contrast, additional fingers do improve results of the position test. No one or two finger configurations were stable with any mispositioning. This is because a grasp with one or two fingers on a sphere becomes unstable as soon as the sphere is moved an incremental distance off center. With this positioning, each of the fingers provides a component of force tending to push the sphere away from finger contact. With increased links and multiple fingers, the sphere is stable until the mispositioning is great enough that the sphere's center is outside the line between two adjacent joints. Then again, the normal forces tend to force the sphere outward and the grasp is unstable. Due to this, the sphere position test shows only slight variations between the $3 / 2$ and the $3 / 3$ configurations.

No single link configuration was stable under any mispositioning due to lack of wrap around. Without wrapping, as the sphere is mispositioned, the normal force vectors gain a component in the direction away from the palm, resulting in instability. Additional links provide the wrapping necessary for restraint. 


\section{The Cube Tests}

The cube size and position tests typically showed little increase in score when fingers or links were increased above two. The scores did not differ significantly between configurations and were primarily determined by the relationship between size of the palm and the size of the cube. In general, most configurations were able to stably grasp a cube of about the same size.

The instability in the cube test typically resulted from the cube's size or mispositioning causing it to actually cover a joint, so that the affected finger could no longer contribute to the grasp. The two finger and four finger configurations gave the same score because the finger base joints became covered at the same time in both the size and position tests. The position test showed the same score in nearly all cases due to the cube moving to a position where it was outside the reach of all fingers. Variations in scores for the one finger configuration are due to differences in performance on the small size portion of the test. For certain proportions, the finger was unable to achieve a wrap around smaller objects due to palm contact on the outer links. Overall, the conclusion to be drawn from the cube test is that grasp stability for a cube in an end effector of this layout is more sensitive to palm size than to finger configuration, provided that the finger joint proportions are adequate to allow wrapping. 


\section{The Cylinder Tests}

Cylinder tests also showed an increase as fingers or links were increased from 1 to 2, with little increase (and sometimes a decrease) as they were further increased. The cylinder size test appeared to rank proportions based on the length of the first link, although the equal length proportions apparently do not follow this trend. We say apparently because the higher scores for the equal length 3 link configuration correspond to the same geometry as the 2 link half length configuration (figure 11). The sets of proportions with the longer base link had typically higher scores on the size test than those with shorter links. 2 link designs were always evaluated to be as good or better than those with 3 links.

3 fingered configurations received lower scores in the size test due to the location of the base joints for fingers 2 and 3 . With a cylinder as pictured in figure 12, the 3 finger configuration placed the second and third finger base joints farther "under" the cylinder than either a 2 or 4 finger configuration. This reduced the ability of the fingers to achieve a wrapped grasp. Like the cube test, the cylinder small size component was responsible for differences in results for the single finger configurations, again because of outer link contact with the palm for certain finger proportions and cylinder sizes.

The cylinder position test showed all single finger and single link configurations unstable for any mispositioning, with the exception of the $1 / 3$ anthropomorphic configuration, which could tolerate only .1 unit of 

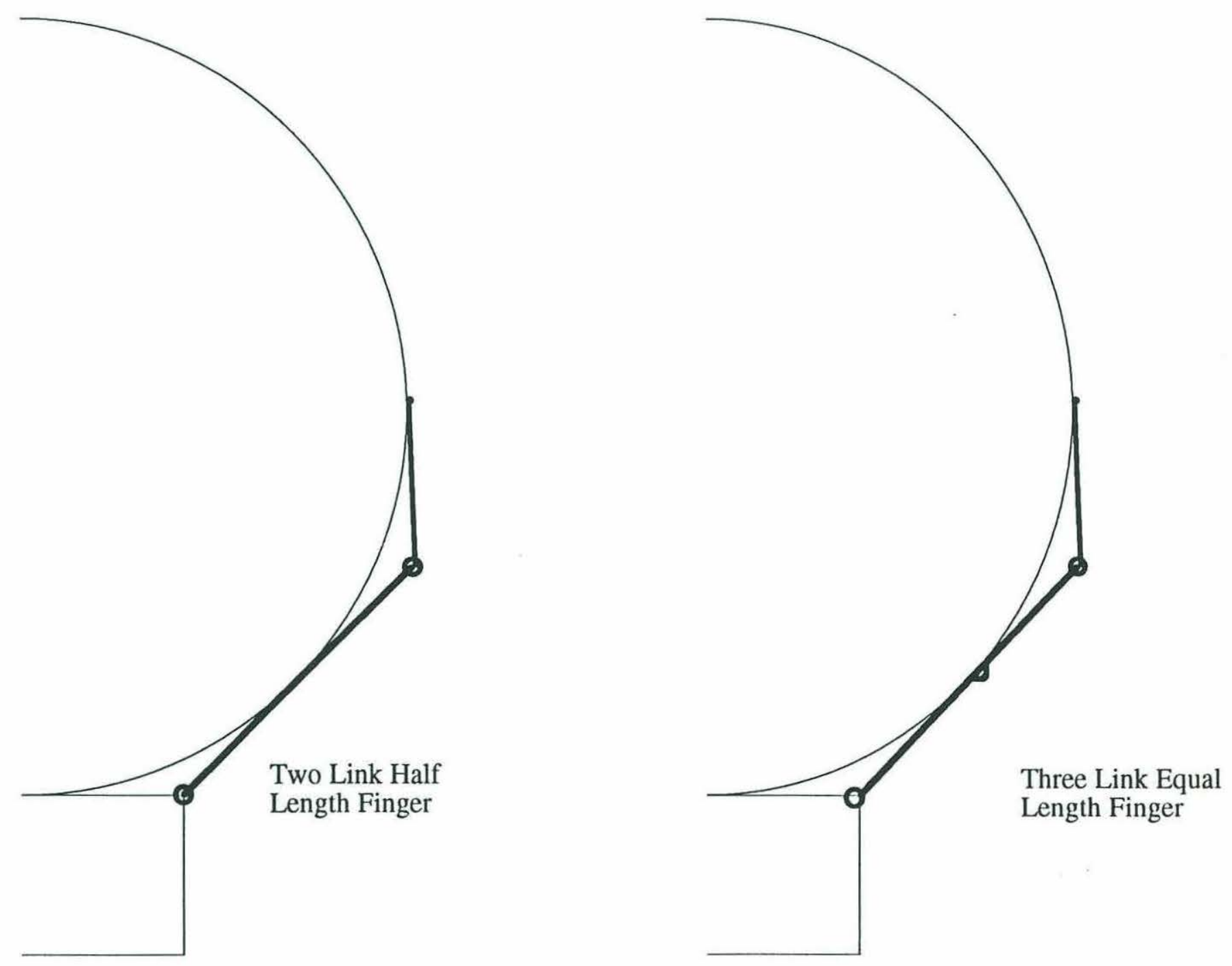

Figure 11: 2 link /half length and 3 link /equal length geometry identical for a circle of radius 2.4 units 

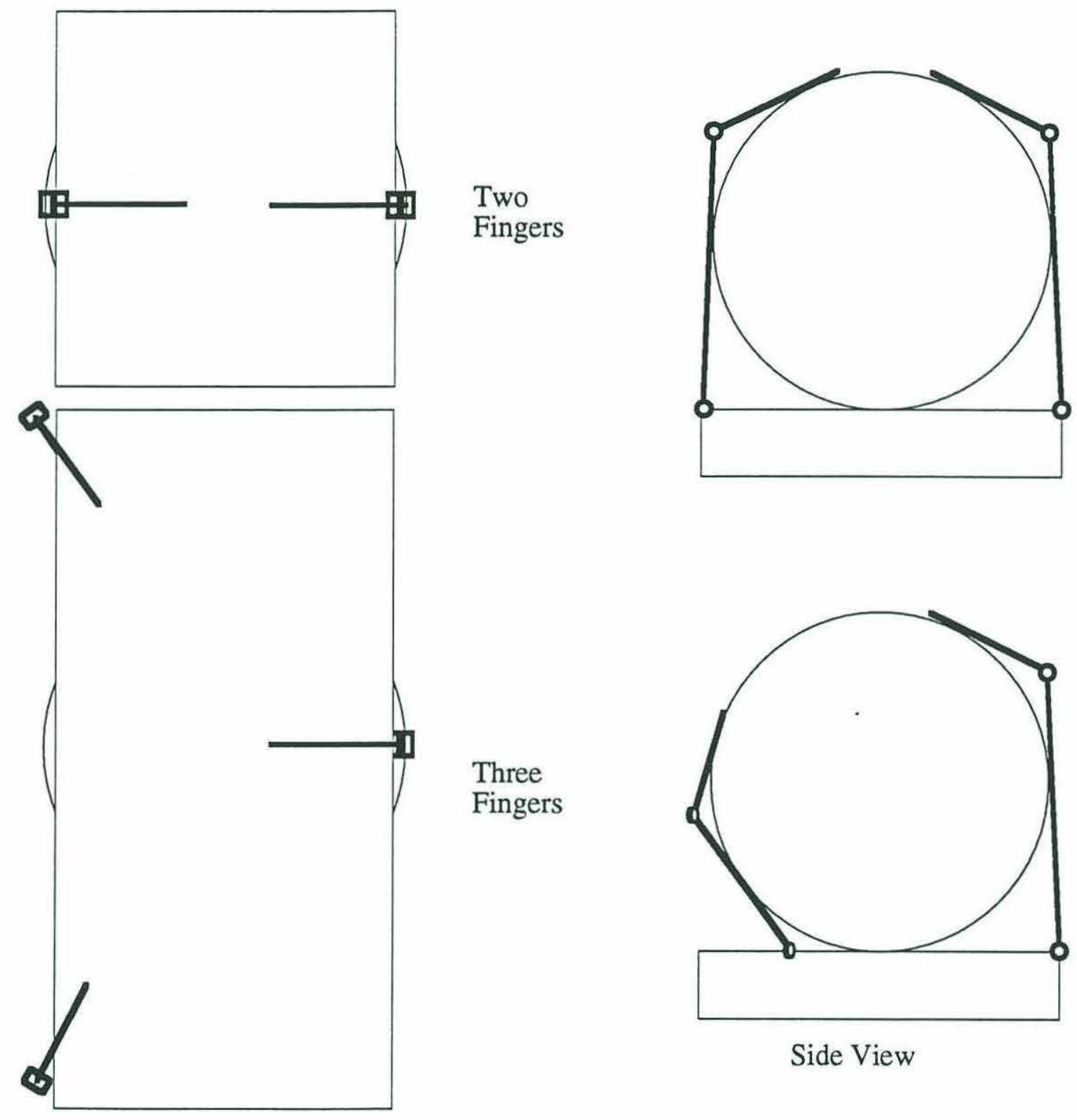

Top View

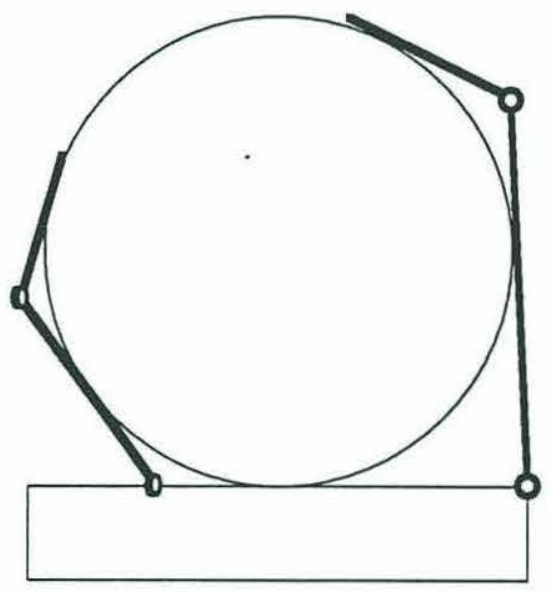

Side View

Figure 12: Location of base joints for 3 finger configuration limits wrapping ability compared to 2 finger configuration 
mispositioning. Again, this configuration's non-zero position score was due to the ability to (barely) wrap around the cylinder even though slightly mispositioned. Each multi-finger, multi-link configuration achieved a similarly high score due to the enhanced wrapping ability and the contribution of other fingers to required contact wrenches.

\section{The 2/2 Half Length Configuration}

The 2/2 configuration with half finger length proportions was selected as the simplest configuration for optimal grasping. The longer base link combined with the shorter second joint allowed significant wrap around capability which typically equalled the grasping ability of the $2 / 3$ configuration with the same proportions. This configuration is unstable, however, when grasping a sphere with any mispositioning. If grasping of spherical shapes was the highest priority, the selected configuration would need to be reevaluated with higher weight given to the sphere test. Alternately, different finger shapes (an example is in Chapter 5) or different finger configurations (two dependent fingers opposing one) could be used. 


\section{Chapter 5}

\section{Model Evaluation and Conclusion}

The previous chapters explained the composition, theory and results of the grasp testing simulation. This chapter discusses model testing and makes some general observations about results of this work. No effort was made to conduct rigorous testing of all prehension possibilities with the model. The computer simulation was a more efficient, reasonable way to do this. Complete testing of all computer results would require a huge selection of test objects in incremental sizes and much more time than required to run the program (currently about four hours on a Sun Sparc 1+ workstation). The model was used to validate results of the computer simulation, as a check to ensure that the numerical results gave accurate and reasonable answers.

\section{Model Validation}

The model was constructed from aluminum rod, strip and angle (figure 13). The palm radius is approximately two inches, giving a model nearly the size of previous end effectors used with the Jason ROV. Additionally, model dimensions could be easily determined by multiplying simulation dimensions by 2 . A set of 1,2 and 3 link fingers was constructed using the half length set 
of proportions recommended by the testing program. We obtained objects which bracketed the largest stable size and used them to conduct

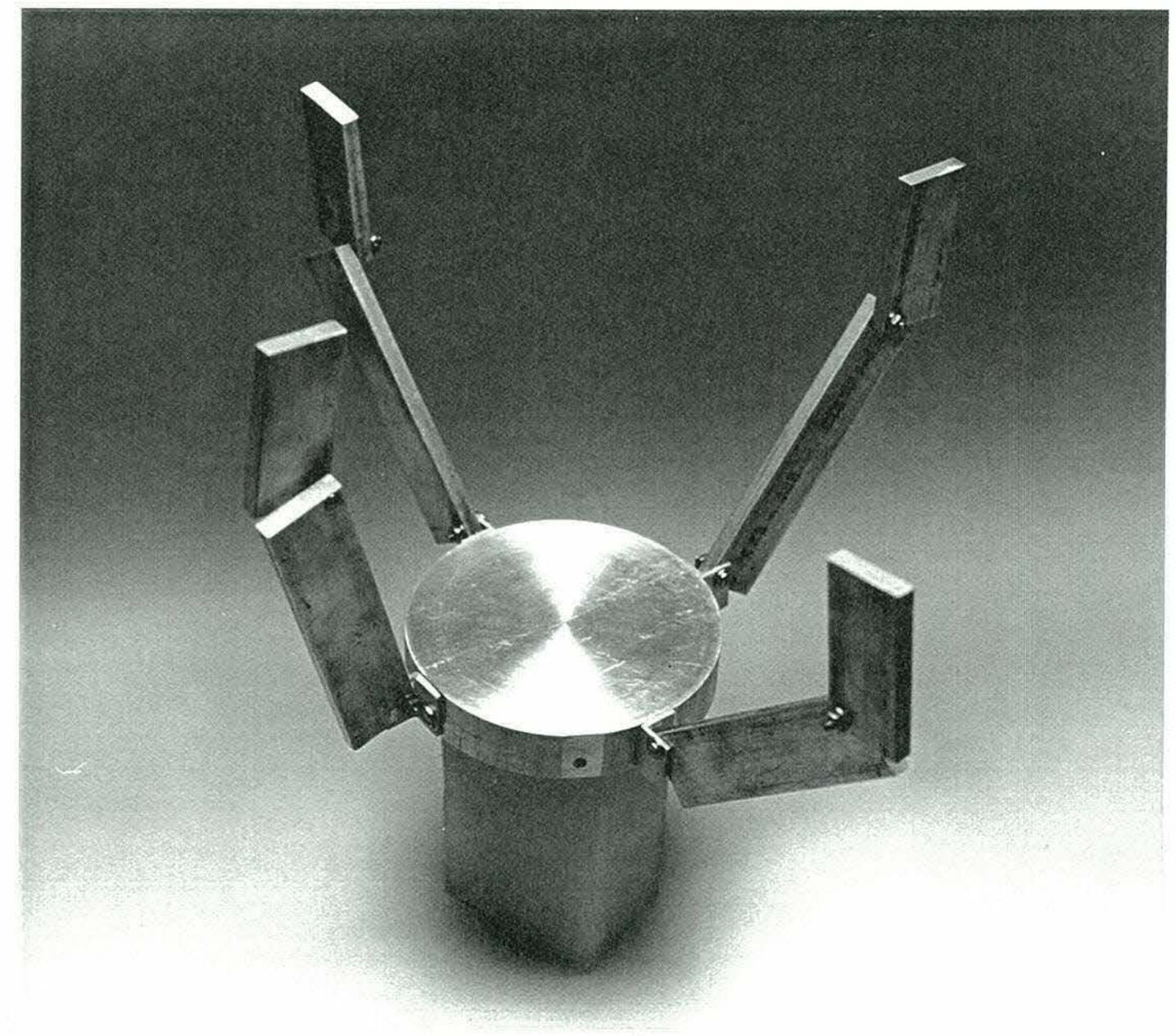

Figure 13: Validation model showing $4 / 2$ configuration

demonstrations of stability and instability.

The demonstrations showed good agreement between simulation results 
and actual grasp stability. Model testing methods were simple. For size testing, the object was centered in the palm and the fingers were closed about it in the appropriate link sequence. The base joint was closed until contact with the test object occurred, then the next joint was closed until contact and so on. Stability was judged based on the ability of the object to move with the finger joints locked in this position.

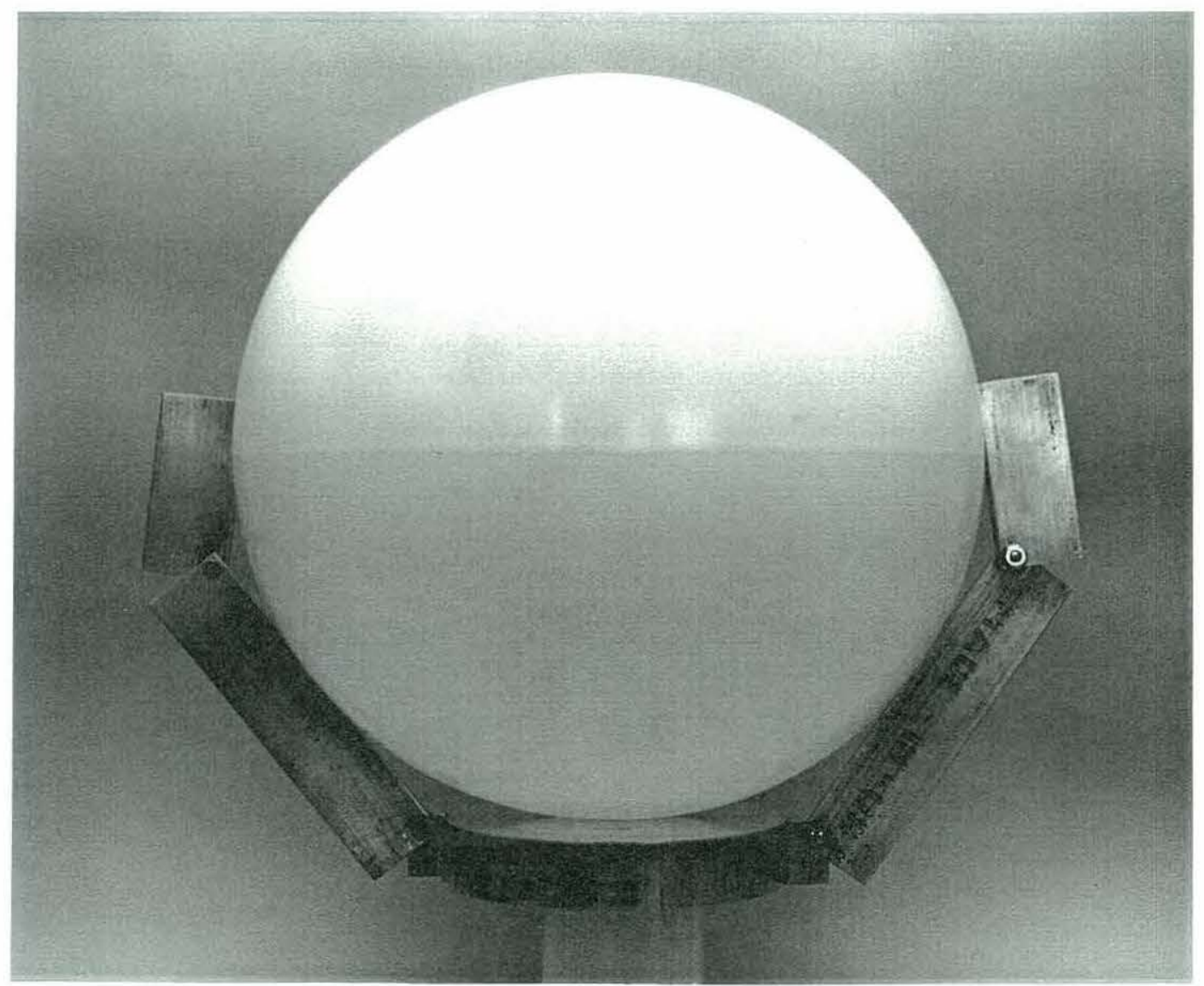

Figure 14: Stable grasp on a sphere with a $2 / 2$ configuration end effector 


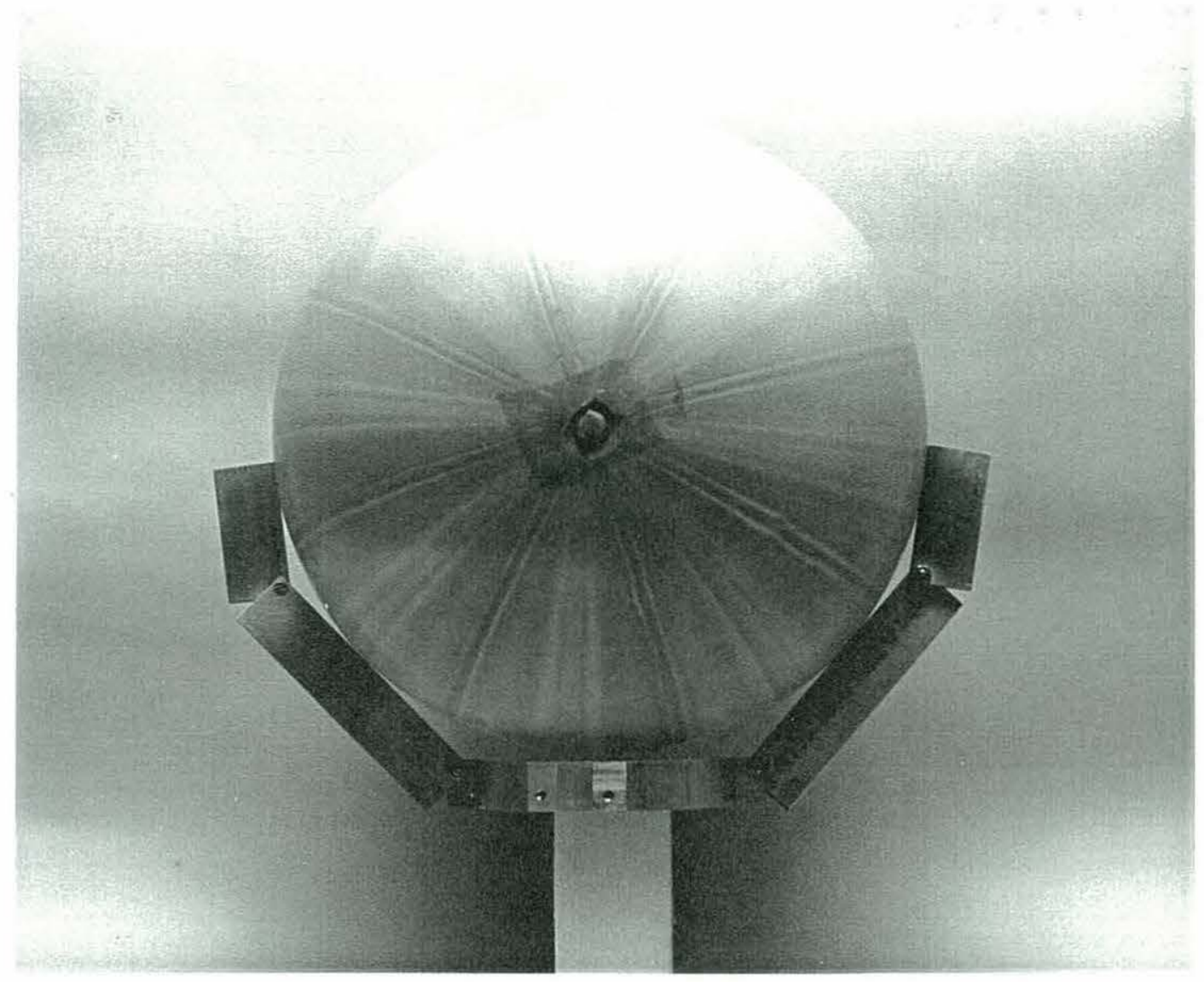

Figure 15: Unstable grasp on a sphere by a $2 / 2$ configuration end effector

For example, consider the grasp shown in figure 14. This figure depicts a sphere size test with a $2 / 2$ hand configuration using half length proportions. The hand is shown grasping a 4.5 inch radius sphere, corresponding to a 2.25 unit radius in the scale of the simulation. The program predicts stability up to a 2.4 unit radius. In the figure, we see that the end links are (barely) able to wrap around the object, contacting the sphere just above its maximum diameter. This provides a stable grasp, as predicted by the simulation. In 
contrast, figure 15 shows the same configuration grasping a 5 inch radius sphere. This corresponds to a 2.5 unit radius object in the program scale and is predicted to be unstable. The end links are unable to extend to the major axis of the sphere. Each normal wrench includes a component directed away from the palm, so the grasp is unable to resist motion in this direction. Any incremental motion of the object away from the palm will cause contact to be broken, resulting in disappearance of all frictional forces. If finger forces were increased, the object would be forced out of the grasp. Addition of 2 more fingers to achieve the $4 / 2$ configuration causes no change in the results of the sphere size test due to the symmetry of the test object.

Figure 16 shows the sphere position test using a sphere of 2 inch radius. As shown in figure 16 , any mispositioning with the $2 / 2$ configuration results in an unstable grasp. Motion in the direction of the finger axes is unresisted if there is any mispositioning in this direction. The region of stability for the position test is considerably expanded, however, when the additional 2 fingers are added to give a $4 / 2$ configuration. These grasps become unstable when mispositioning allows the sphere to be unrestrained against motion in the direction diagonal to the 2 fingers (in the direction of the mispositioning). This is shown in figure 17, where the fourth finger has been omitted for clarity. Though not considered in the simulation, the model design allows finger collisions for this grasp.

The cylinder size test for configurations with left/right symmetry gave 


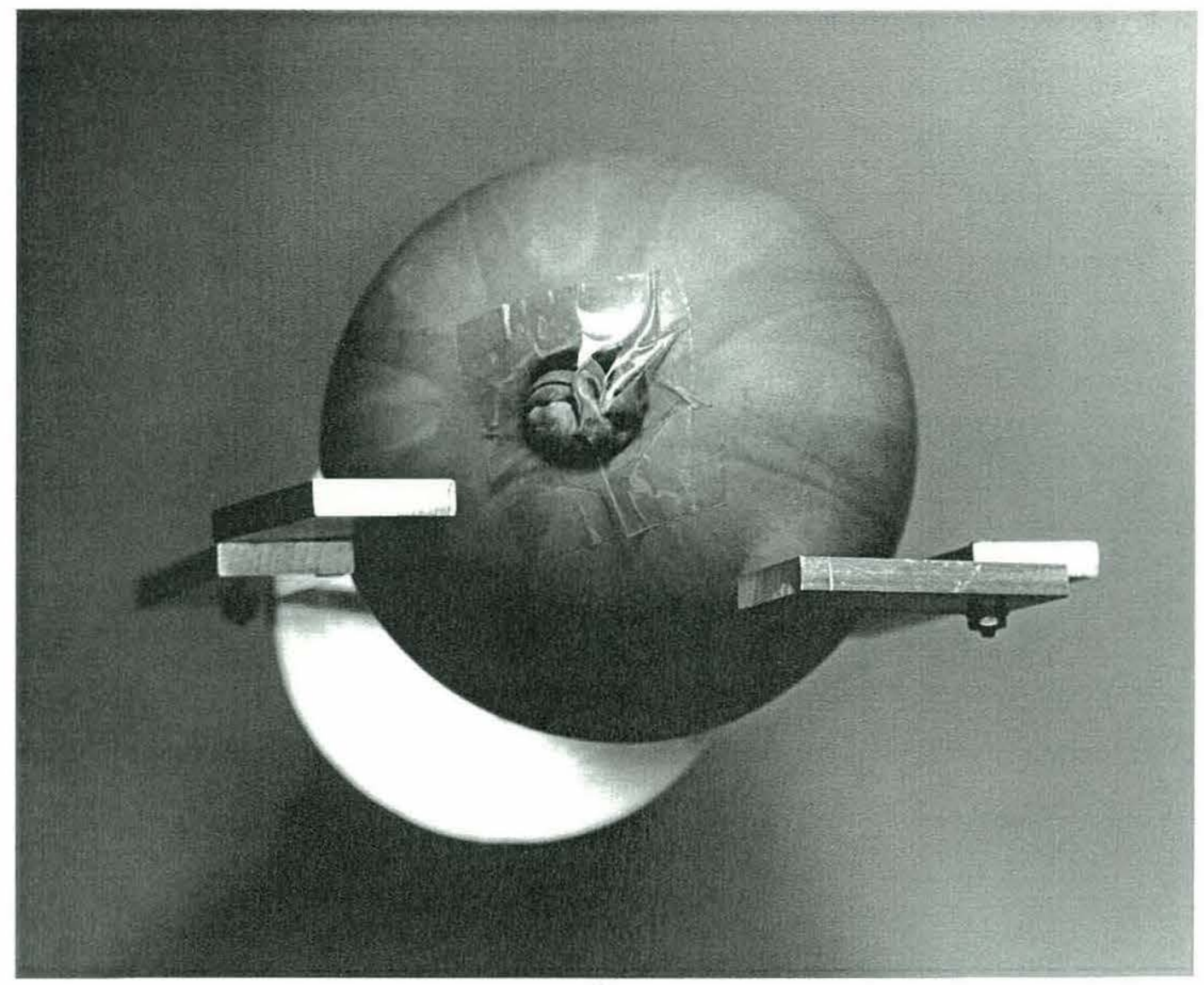

Figure 16: Sphere position test with $2 / 2$ configuration end effector results identical to that of the sphere since the two dimensional representation of each is identical in the plane of the fingers. The test for three fingered configurations generally verified results of the simulation. Figure 18 shows a $3 / 2$ configuration grasping a 7 inch diameter cylinder. This is below the predicted stability limit of 7.2 inches (1.8 radius in the simulation scale). The second link contacts wrap around to give contact wrenches located on the far 


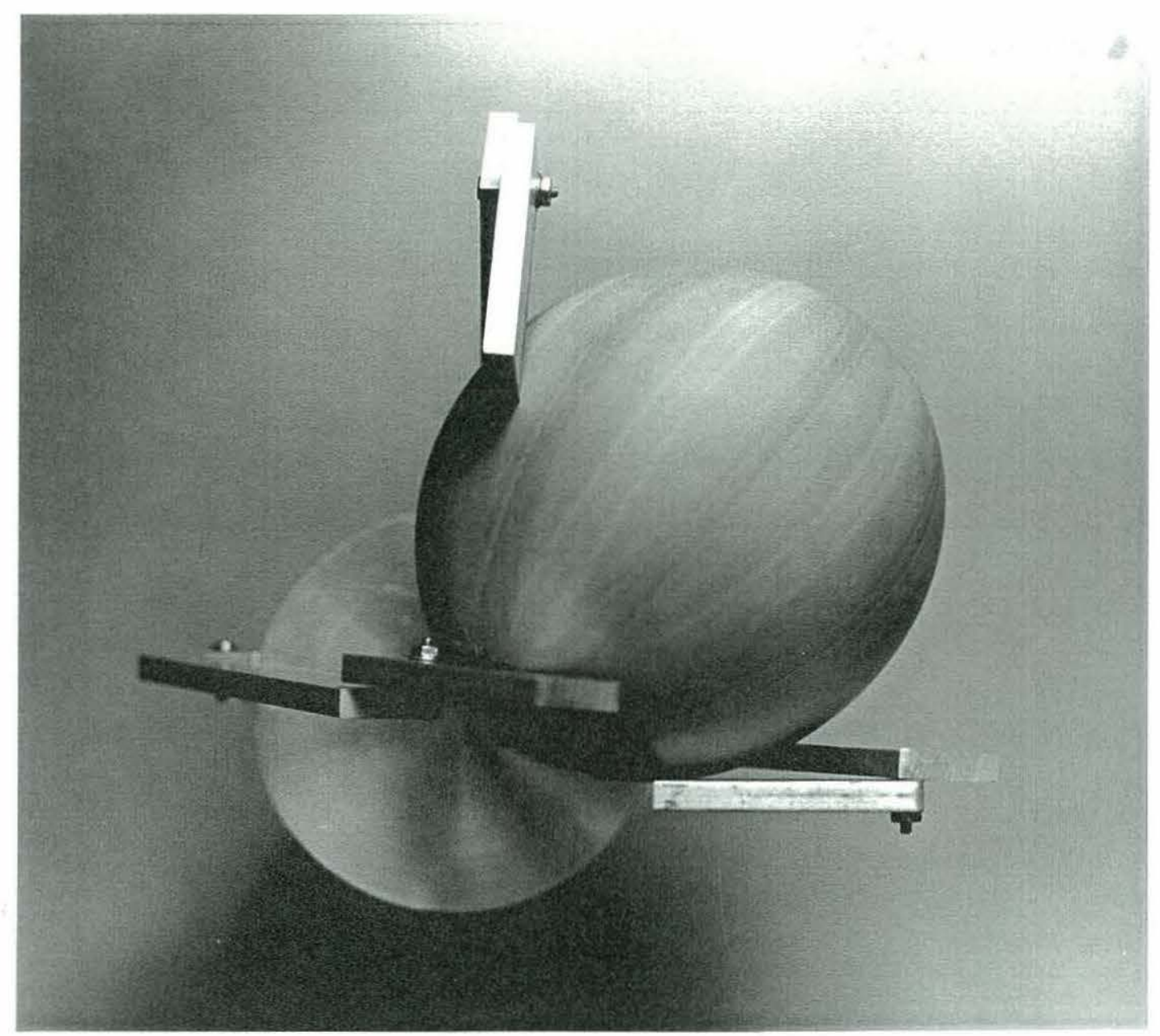

Figure 17: Sphere position test with 4/2 configuration end effector, fourth finger removed for clarity

side of the maximum diameter, resulting in stable grasp. The largest stable size for 3 fingered configurations is smaller than for 2 fingered configurations, due to the greater effective distance between the finger base joints in the 2 fingered case. The 3 finger configuration gives a smaller perpendicular distance between fingers, limiting the ability to wrap around larger objects. 


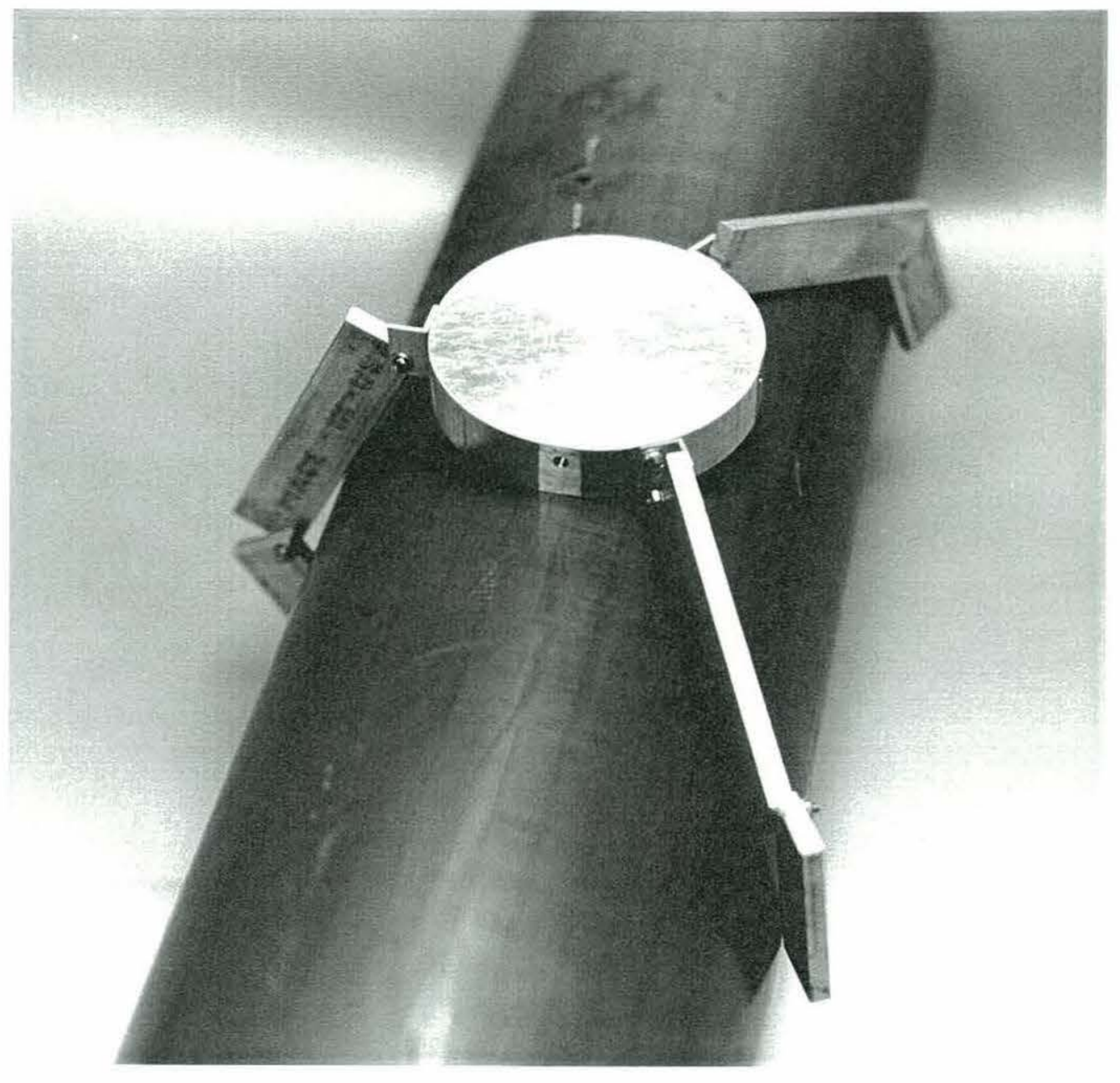

Figure 18: Stable grasp on a cylinder by a $3 / 2$ end effector

Figure 19 shows a cylinder position test of a $2 / 2$ configuration end effector grasping a cylinder near but within the stability limit. The cylinder pictured is of 2.25 inch radius, roughly corresponding to the size of the object used in the simulation's position test. The deviation is due to small departures from ideal dimensions present in the model. Although the palm is 2 inches in 


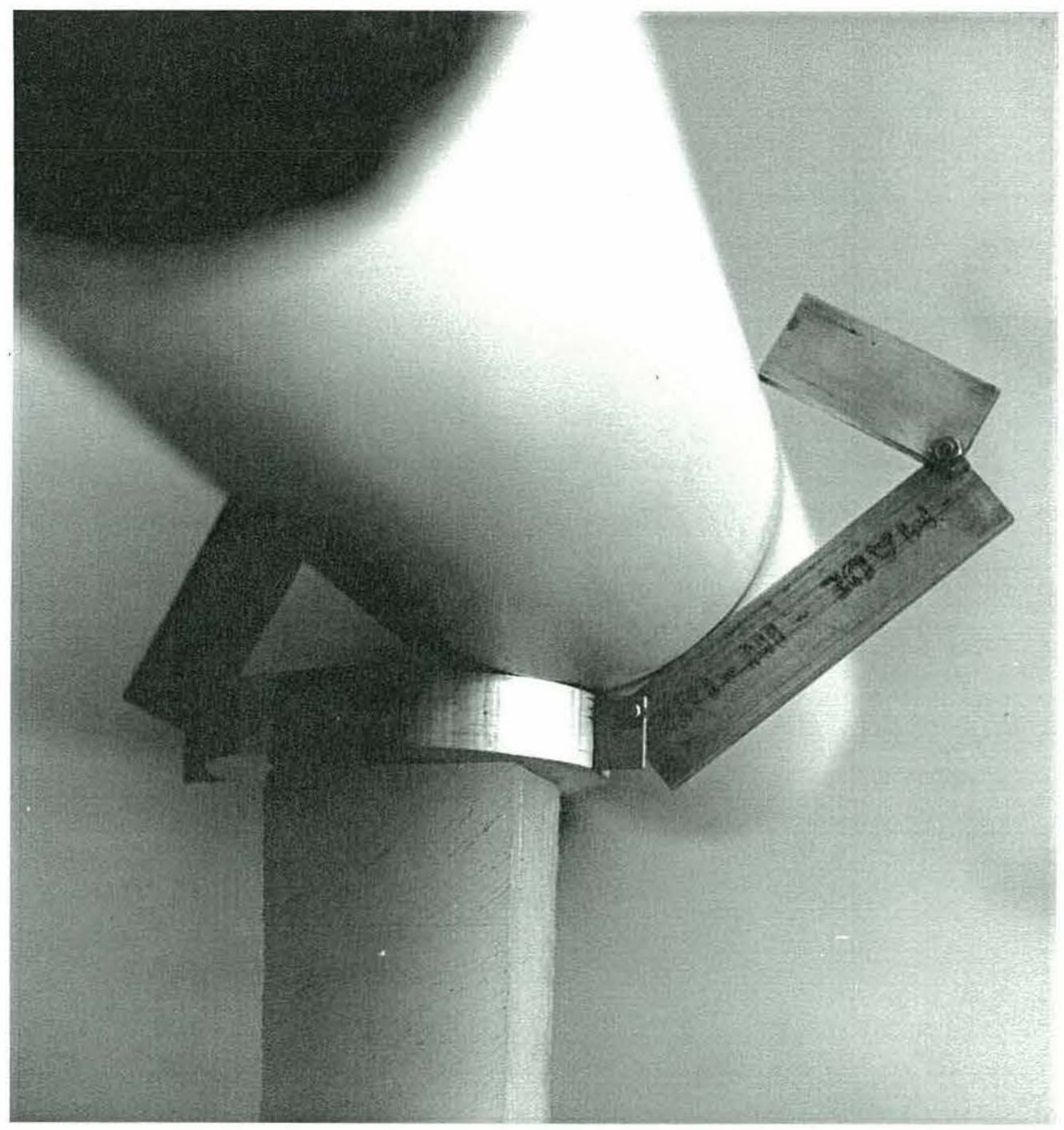

Figure 19: Stable grasp on a cylinder by a $2 / 2$ end effector radius, physical restrictions on the finger brackets required placement of the base joints at a 2.25 inch radius. We find that even with the mispositioning, the grasp is stable. However, if we increase the mispositioning by a small amount (figure 20), contact is lost on the last link of the right side finger. 


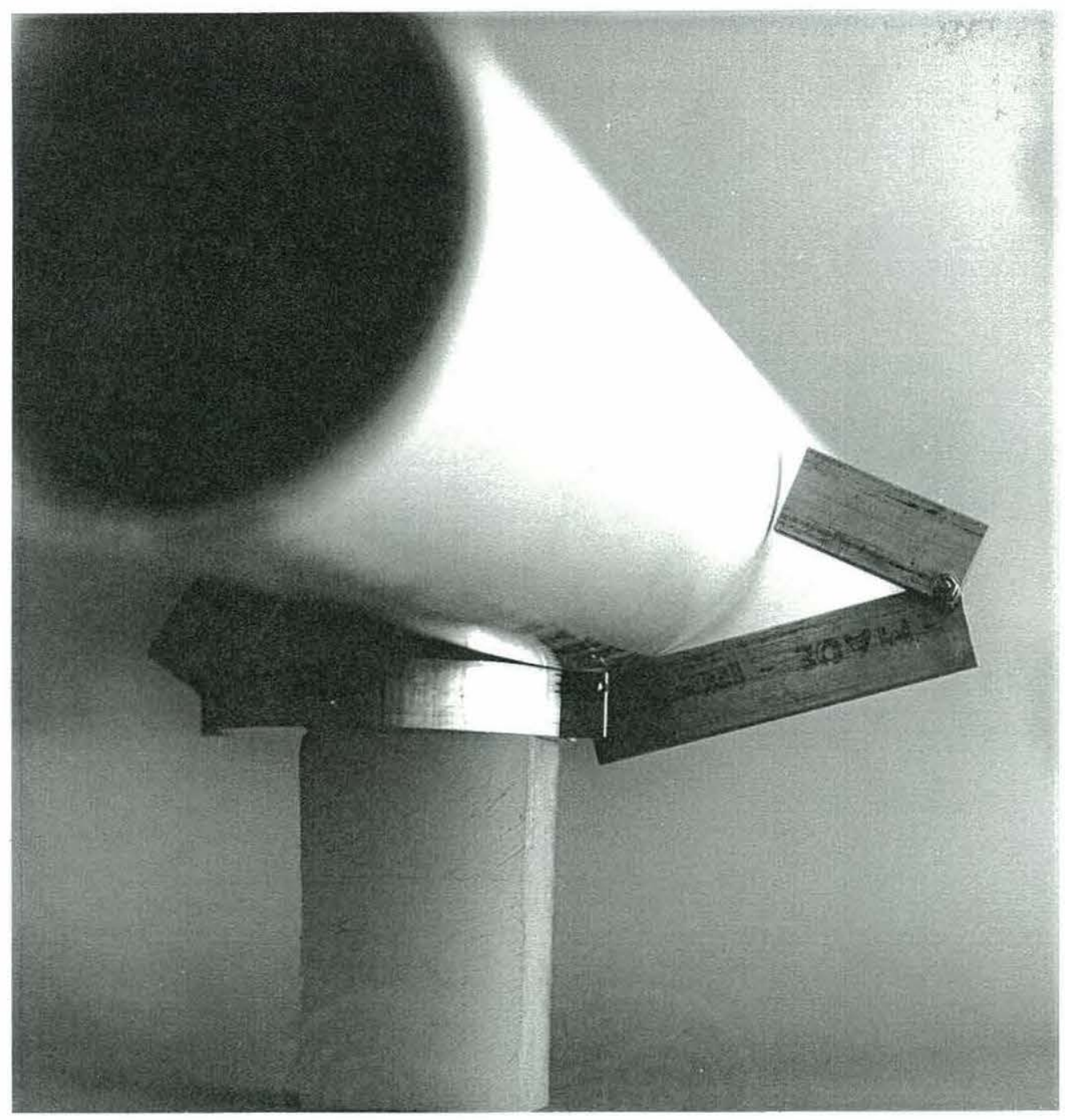

Figure 20: Unstable cylinder position test for a $2 / 2$ end effector

Without the contribution from this link, the object is unrestrained from motion diagonally up and to the right. These figures correspond to a mispositioning of .8 units for figure 18 and .9 units for figure 19, within .1 of the values determined by the testing program. Deviations are due to departures from 
ideal dimensions and inaccuracies in measuring positioning in the model.

A demonstration of the size test was also done using a cube of dimension 4 inches on a side. This corresponds to an approximate 2 unit side length for the simulation and is near the predicted largest stable size for each configuration and proportion. As previously discussed, cube stability relates to palm radius rather than to finger and joint configuration. The slightly smaller size of 1.7 units for the 3 finger configurations is due to the decreased linear distance between the second two finger's base joints. 1.7 units is simply the largest cube size that will fit between these joints. Because of this, we did not attempt a cube position test using the model. The cube position results again reflected palm size instead of configuration. The result of .5 units represented the mispositioning beyond which fingers could no longer reach the cube or at which joints became covered.

We must point out that grasps near the limit of stability are just that - possible grasps near the limit of stability. No attempt was made to characterize grasps with respect to quality. The actual largest size object which could be stably grasped is likely to be smaller than that predicted by the grasp tester, primarily due to our treatment of friction. As noted before, no attempt was made to deal with the magnitude of frictional forces. For an actual grasper and for a given coefficient of friction, as the limit of stability is approached, increasing finger forces would be required to prevent slipping of the object. The required forces may be larger than a given end effector could 
exert. Additionally, finger compliance would cause the intersection points to deviate from those the simulation predicts for rigid fingers.

Since our goal was to generate a ranked comparison of various configurations and proportions, the above deviations are acceptable. The implicit assumption is that the ranking of finger configurations and proportions will remain invariant when transferred to an actual grasper. The largest size and position values are likely to be somewhat less, but the relative grasping ability should remain the same. While this cannot be proven, it is intuitively reasonable. The proof would require consideration both of limiting finger forces, finger compliance and frictional force magnitudes which are beyond the scope of this work.

\section{Observations}

Simulation results and model validation lead us to several simple observations on the choice of end effector configurations. These observations are strictly valid only for the specific hand assumptions considered in this work, however, they form a set of straightforward guidelines for general design of an end effector.

If the goal of the end effector is to stably grasp rectangular objects, the palm dimensions and distance between base finger joints are more important than finger configurations or proportions. This is because of an explicit assumption of the grasp tester which is patterned after real world 
expectations; if a finger joint is covered by the object, the grasp will fail. If the joint is covered, the obscured finger essentially becomes an extension of the palm, unless it is incapable of bending "backwards". All possible finger contacts with the obscured finger are lost except for those duplicating palm contact wrenches. To prevent this, the palm diameter must be greater than or equal to the cross section of the largest rectangular object to be grasped.

If the objective is stable grasping of a cylindrical object, the largest size object is affected both by the palm diameter and finger and link configuration. Assuming that finger length is increased in proportion to palm size, wider palms and longer fingers promote stable grasping of larger objects. Finger configurations that maximize distance between opposing finger base joints promote stable grasping for larger objects. The key to a stable grasp on a cylinder is the ability to provide finger contacts at or above the widest effective diameter of the cylinder. Multi-jointed fingers increase the size of cylinders that can be stably grasped due to their ability to wrap around the cylinder. A 3 jointed finger typically can not wrap as far around an object as a 2 jointed finger. This is not obvious. Reference to figure 21 illustrates this result. The figure shows a circle of radius 2.4 units and fingers with both 2 and 3 links of half length proportions. The contact from the second link of the 3 link finger does not add significantly to stability since it lies below the maximum diameter; it is redundant to the first joint contact. The contact from the third link occurs at the point of tangency between the circle and that link. Thus, it 


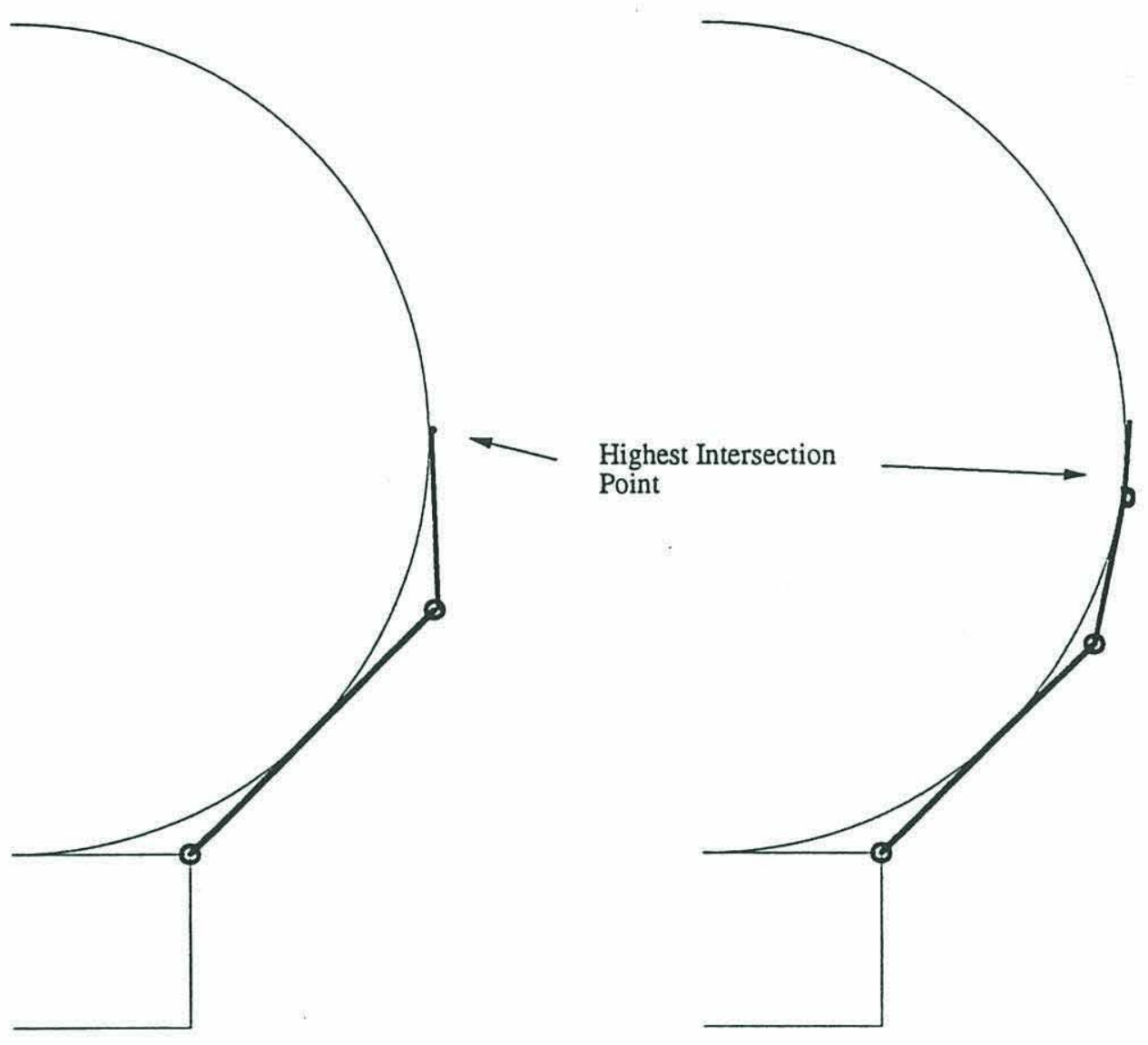

Figure 21: Comparison of wrapping abilities for 2 and 3 link fingers of half length proportions.

does not take advantage of the full length of the finger. In contrast, the 2 link finger's final contact occurs at the tip of the last link. The geometry of the 2 
link configuration brings the second joint to a position so that the last link cannot reach the tangent point. This drives the last link into making tip contact, effectively using more of the finger's length. With a good choice of finger proportions, 2 link fingers can allow stable grasping of as large (or larger) objects than 3 link fingers.

One case examined did show better grasping scores for the 3 link configuration than for 2 links. The equal proportioned 3 link finger's sphere and cylinder size scores were higher than the 2 link scores (Appendix I). A grasp by a 3 link finger on a circle of radius 2.4 units reveals a geometry identical to that of the half proportion 2 link finger (figure 11). The largest score achieved by a 3 link finger occurred when the finger assumed the geometry of a 2 link finger!

As noted in Chapter 4, the 2 finger 2 link configuration appears to provide an optimum balance of minimum complexity with grasp performance. The only drawback to this configuration is the instability of grasps on spherical objects which are not aligned with the center of the palm. This drawback can be minimized by use of finger shape or configuration to expand the region of stability. The simulation assumed the fingers were simple lines, however providing wide fingers with concave contact surfaces would allow stable grasping of spheres as long as the center was within the outer limits of the sides of the fingers. Figure 22a shows an overhead view of a possible finger shape that would enhance the region of stability for this grasp. The Penn 

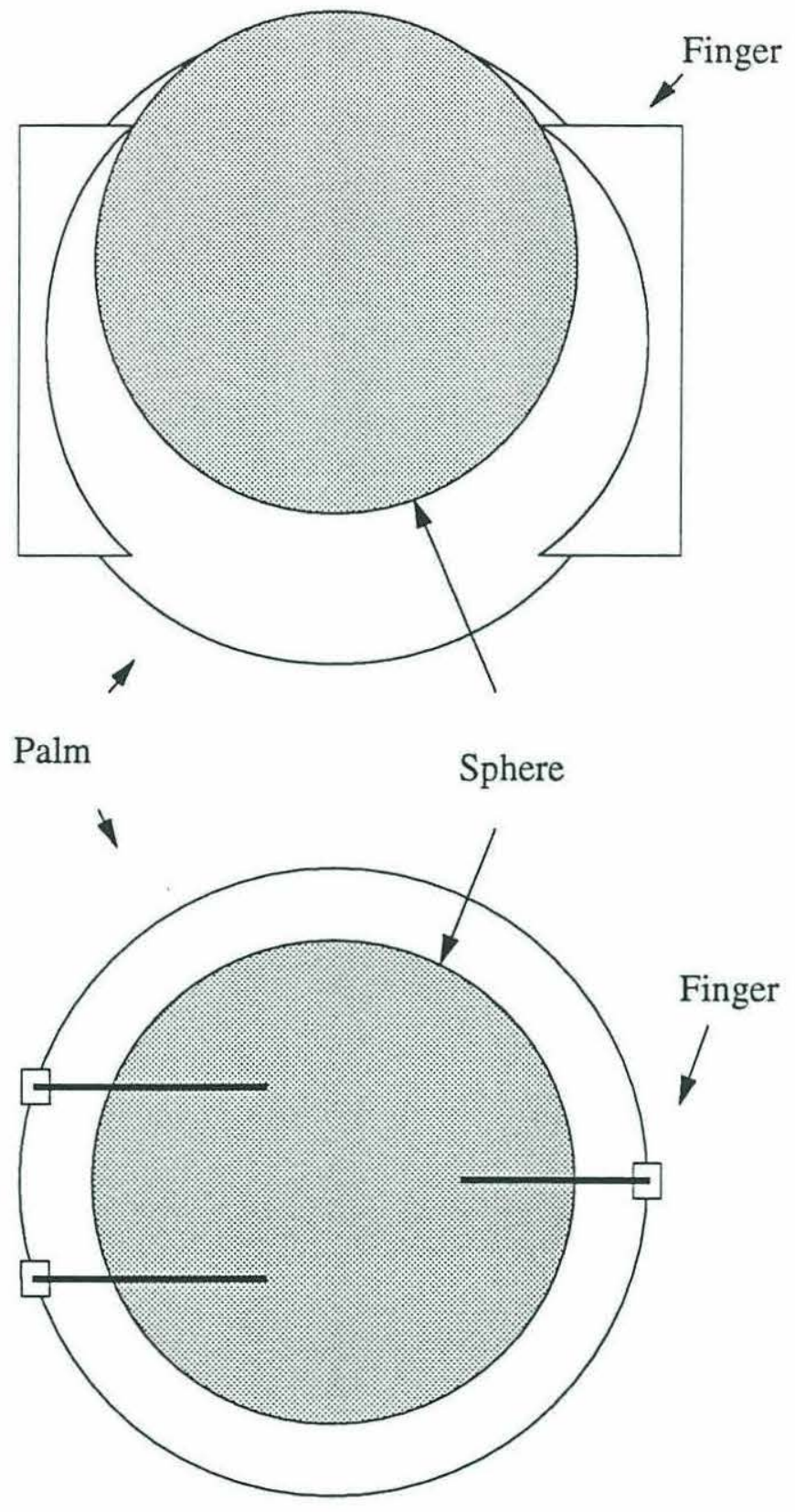

Figure 22: Methods of enhancing sphere stability: a)Finger shape b) Configuration and orientation 
Hand can effect a configuration where 2 fingers oppose a single finger (figure 22b). This also improves grasp stability on a sphere or cylinder and could be incorporated into a simpler end effector. 
Appendix 1: Grasp Tester Results 
Grasp Tester Results for Half Length Proportions

SPHERE

Links

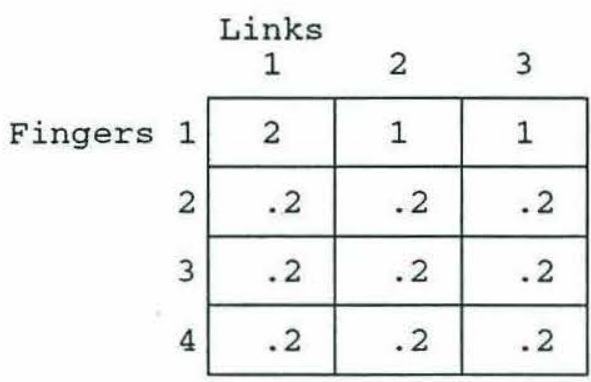

Links

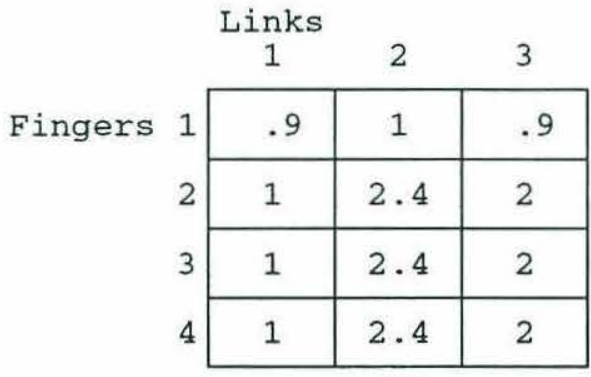

Links

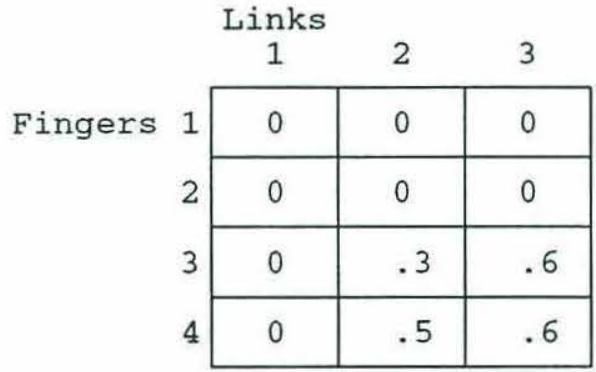

CUBE

CYLINDER

Small Size Test

\begin{tabular}{|l|l|l|}
\multicolumn{1}{c}{1} & \multicolumn{1}{l}{2} & 3 \\
\hline 1 & .4 & .6 \\
\hline .2 & .2 & 2 \\
\hline .2 & .2 & .2 \\
\hline .2 & .2 & .2 \\
\hline
\end{tabular}

\begin{tabular}{|l|l|l|}
\multicolumn{1}{l}{1} & \multicolumn{1}{l}{2} & 3 \\
\hline .5 & .1 & .3 \\
\hline .2 & .2 & .2 \\
\hline .2 & .2 & .2 \\
\hline .2 & .2 & .2 \\
\hline
\end{tabular}

Large Size Test

\begin{tabular}{|c|c|c|}
\multicolumn{1}{c}{1} & \multicolumn{1}{c}{2} & 3 \\
\hline .9 & 1.9 & 1.7 \\
\hline 1.9 & 1.9 & 1.9 \\
\hline 1.7 & 1.7 & 1.7 \\
\hline 1.9 & 1.9 & 1.9 \\
\hline
\end{tabular}

\begin{tabular}{|l|l|l|}
\multicolumn{1}{l}{1} & \multicolumn{1}{l}{2} & 3 \\
\hline .4 & 1 & .9 \\
\hline 1 & 2.4 & 2 \\
\hline .7 & 1.8 & 1.7 \\
\hline 1 & 2.4 & 2 \\
\hline
\end{tabular}

Position Test

\begin{tabular}{|l|l|l|}
\multicolumn{1}{l}{1} & \multicolumn{1}{l}{2} & 3 \\
\hline 0 & .4 & .5 \\
\hline .5 & .5 & .5 \\
\hline 0 & .5 & .5 \\
\hline .5 & .5 & .5 \\
\hline
\end{tabular}

\begin{tabular}{|c|c|c|}
\multicolumn{1}{l}{1} & 2 & 3 \\
\hline 0 & 0 & 0 \\
\hline 0 & .7 & 1 \\
\hline 0 & .8 & 1 \\
\hline 0 & .7 & 1 \\
\hline
\end{tabular}

Links

Raw Total Score

Normalized Total Score

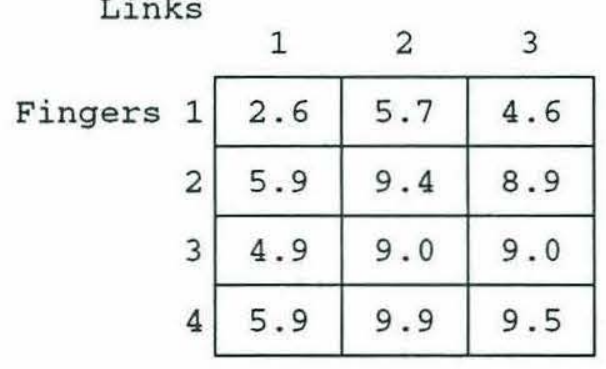

\begin{tabular}{|c|c|c|}
\multicolumn{1}{c}{1} & \multicolumn{1}{c}{2} & 3 \\
\hline 1.0 & 2.8 & 2.5 \\
\hline 3.0 & 4.7 & 4.7 \\
\hline 1.8 & 5.0 & 5.5 \\
\hline 3.0 & 5.5 & 5.7 \\
\hline
\end{tabular}


Grasp Tester Results for Equal Length Proportions

\begin{tabular}{|c|c|c|c|c|}
\hline & \multicolumn{3}{|c|}{ SPHERE } \\
\hline & & \multicolumn{3}{|c|}{ Links } \\
\hline & & 1 & 2 & 3 \\
\hline Fingers & 1 & 2 & 1 & 1 \\
\hline & 2 & .2 & .2 & .2 \\
\hline & 3 & .2 & .2 & .2 \\
\hline & 4 & .2 & .2 & .2 \\
\hline
\end{tabular}

CUBE

CYLINDER

\begin{tabular}{c|c|c|c|c|} 
& \multicolumn{4}{c}{ Links } \\
\cline { 3 - 5 } Fingers & 1 & \multicolumn{1}{c}{2} & 3 \\
\cline { 2 - 5 } & 2 & .9 & .9 & 1 \\
\cline { 2 - 5 } & 3 & 1 & 1.7 & 2.4 \\
\cline { 2 - 5 } & 4 & 1 & 1.7 & 2.4 \\
\cline { 2 - 5 } & 1 & 1.7 & 2.4 \\
\hline
\end{tabular}

Small Size Test

\begin{tabular}{|c|c|c|}
\multicolumn{1}{c}{1} & 2 & 3 \\
\hline 1 & .4 & .2 \\
\hline .2 & .2 & .2 \\
\hline .2 & .2 & .2 \\
\hline .2 & .2 & .2 \\
\hline
\end{tabular}

\begin{tabular}{|c|c|c|}
\multicolumn{1}{c}{1} & 2 & 3 \\
\hline .5 & .3 & .3 \\
\hline .2 & .2 & .2 \\
\hline .2 & .2 & .2 \\
\hline .2 & .2 & .2 \\
\hline
\end{tabular}

Large Size Test

\begin{tabular}{|r|r|r|}
\multicolumn{1}{c}{1} & 2 & 3 \\
\hline .9 & 1.4 & 1.9 \\
\hline 1.9 & 1.9 & 1.9 \\
\hline 1.7 & 1.7 & 1.7 \\
\hline 1.9 & 1.9 & 1.9 \\
\hline
\end{tabular}

\begin{tabular}{|c|c|c|}
\multicolumn{1}{c}{1} & \multicolumn{1}{c}{2} & 3 \\
\hline .4 & .7 & 1 \\
\hline 1 & 1.7 & 2.4 \\
\hline .7 & 1.4 & 1.4 \\
\hline 1 & 1.7 & 2.4 \\
\hline
\end{tabular}

\begin{tabular}{c|c|c|c|c|} 
& & \multicolumn{3}{c}{ Links } \\
Fingers 1 & 1 & 2 & 3 \\
\cline { 2 - 5 } & & 0 & 0 & 0 \\
\cline { 2 - 5 } & 2 & 0 & 0 & 0 \\
\cline { 2 - 5 } & 3 & 0 & .3 & .4 \\
\cline { 2 - 5 } & & 0 & .5 & .6 \\
\hline
\end{tabular}

Position Test

\begin{tabular}{|c|c|c|}
\hline 1 & \multicolumn{1}{c}{2} & \multicolumn{1}{l}{3} \\
\hline 0 & .5 & .5 \\
\hline .5 & .5 & .5 \\
\hline 0 & .5 & .5 \\
\hline .5 & .5 & .5 \\
\hline
\end{tabular}

\begin{tabular}{|l|l|l|}
\multicolumn{1}{l}{1} & \multicolumn{1}{l}{2} & 3 \\
\hline 0 & 0 & 0 \\
\hline 0 & 1 & 1 \\
\hline 0 & 1 & 1 \\
\hline 0 & 1 & 1 \\
\hline
\end{tabular}

Raw Total Score

Normalized Total Score

Links

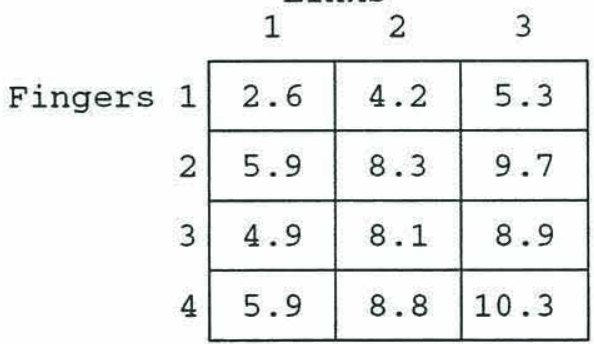

\begin{tabular}{|c|c|c|}
\multicolumn{1}{c}{1} & \multicolumn{1}{c}{2} & 3 \\
\hline 1.0 & 2.4 & 2.8 \\
\hline 3.0 & 4.5 & 5.0 \\
\hline 1.8 & 4.8 & 5.2 \\
\hline 3.0 & 5.4 & 6.0 \\
\hline
\end{tabular}


Grasp Tester Results for Anthropomorphic Proportions

SPHERE

\section{Links}

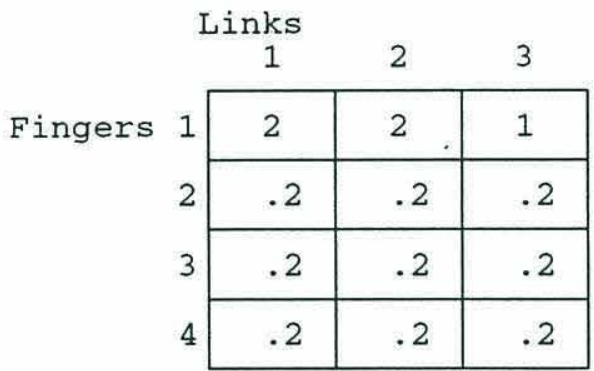

\section{Links}

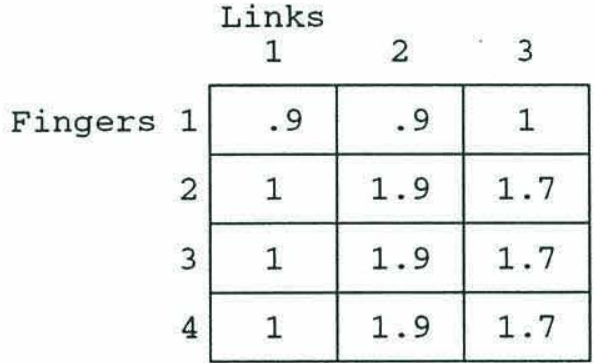

\section{Links}

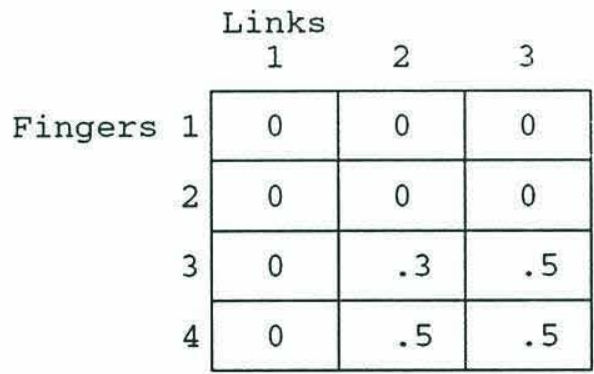

CUBE

Small Size Test

\begin{tabular}{|l|l|l|}
\multicolumn{1}{|l}{1} & \multicolumn{1}{l}{2} & 3 \\
\hline 1 & .4 & .4 \\
\hline .2 & .2 & .2 \\
\hline .2 & .2 & .2 \\
\hline .2 & .2 & .2 \\
\hline
\end{tabular}

Large Size Test

\begin{tabular}{|c|c|c|}
\hline \multicolumn{1}{c}{1} & \multicolumn{1}{c}{2} & 3 \\
\hline .9 & 1.6 & 1.3 \\
\hline 1.9 & 1.9 & 1.9 \\
\hline 1.7 & 1.7 & 1.7 \\
\hline 1.9 & 1.9 & 1.9 \\
\hline
\end{tabular}

\begin{tabular}{|c|c|c|}
\multicolumn{1}{c}{1} & \multicolumn{1}{l}{2} & 3 \\
\hline .4 & .8 & 1 \\
\hline 1 & 1.9 & 1.7 \\
\hline .7 & 1.5 & 1.6 \\
\hline 1 & 1.9 & 1.7 \\
\hline
\end{tabular}

Position Test

\begin{tabular}{|l|l|l|}
\multicolumn{1}{l}{1} & \multicolumn{1}{l}{2} & 3 \\
\hline 0 & .5 & .5 \\
\hline .5 & .5 & .5 \\
\hline 0 & .5 & .5 \\
\hline .5 & .5 & .5 \\
\hline
\end{tabular}

\begin{tabular}{|l|l|l|}
\multicolumn{1}{l}{1} & \multicolumn{1}{c}{2} & 3 \\
\hline 0 & 0 & .1 \\
\hline 0 & 1 & 1 \\
\hline 0 & 1 & 1 \\
\hline 0 & 1 & 1 \\
\hline
\end{tabular}

Raw Total Score

Normalized Total Score

Links

\begin{tabular}{|c|c|c|c|c|}
\hline & & 1 & 2 & 3 \\
\hline \multirow[t]{4}{*}{ Fingers } & 1 & 2.6 & 4.4 & 5.3 \\
\hline & 2 & 5.9 & 8.7 & 8.3 \\
\hline & 3 & 4.9 & 8.4 & 8.5 \\
\hline & 4 & 5.9 & 9.2 & 8.8 \\
\hline
\end{tabular}

CYLINDER

\begin{tabular}{|c|c|c|}
\multicolumn{1}{c}{1} & \multicolumn{1}{c}{2} & 3 \\
\hline .5 & .3 & .1 \\
\hline .2 & .2 & .2 \\
\hline .2 & .2 & .2 \\
\hline .2 & .2 & .2 \\
\hline
\end{tabular}




\section{References}

Abel, J.M., Holzman, W., and McCarthy, J.M., "On Grasping Planar Objects with Two Articulated Fingers", IEEE Conference on Robotics and Automation, 1985.

Ball, R. S., "A Treatise on the Theory of Screws", Cambridge University Press, Cambridge, 1900.

Gale, D., "The Theory of Linear Economic Models", McGraw-Hill, 1960.

Greiner, H., "Passive and Active Grasping with a Prehensile Robot EndEffector", Master's Thesis, Mass. Inst. of Tech., May 1990.

Hanafusa, H. and Asada, H., "Stable Prehension by a Robot Hand with Elastic Fingers", Proc. 7th Int. Symp. on Industrial Robots, Tokyo, October, 1977, pp. 361-368.

Hunt, K. H., "Kinematic Geometry of Mechanisms", Clarendon, Oxford, 1978.

Kuhn, H.W. and Tucker, A.W., editors, "Linear Inequalities and Related Systems", Annals of Math. Studies, Vol. 38, Princeton, 1956, pp. 19-39.

Lakshminarayana, K., "Mechanics of Form Closure", ASME paper No. 78DET-32, 1978.

Nguyen, V, "Constructing Force-Closure Grasps", IEEE Conference on Robotics and Automation, 1986.

Nguyen, V, "Constructing Force-Closure Grasps in 3D", IEEE Conference on Robotics and Automation, 1987a.

Nguyen, V, "Constructing Stable Grasps in 3D", IEEE Conference on Robotics and Automation, 1987b.

Ohwovoriole, M.S., "Kinematics and Friction in Grasping by Robotic Hands", 
Journal of Mechanisms, Transmissions, and Automation in Design, Vol. 109, pg. 398, Sept 1987.

Ohwovoriole, M.S. and Roth, B., "An Extension of Screw Theory", Journal of Mechanical Design, Vol. 103, pg. 725, Oct 1981.

Salisbury, J.K., and Mason, M.T., "Robot Hands and the Mechanics of Manipulation", MIT Press, Cambridge, Massachusetts, 1985.

Trinkle, J.C., "The Mechanics and Planning of Enveloping Grasps", Phd Dissertation, U. of Penn., 1987.

Ulrich, N.T., "Grasping with Mechanical Intelligence", Master's Thesis, U. Penn., Philadelphia, Dec., 1988. 